TRANSACTIONS OF THE

AMERICAN MATHEMATICAL SOCIETY

Volume 348, Number 6, June 1996

\title{
SMOOTH CLASSIFICATION OF GEOMETRICALLY FINITE ONE-DIMENSIONAL MAPS
}

\author{
YUNPING JIANG
}

\begin{abstract}
The scaling function of a one-dimensional Markov map is defined and studied. We prove that the scaling function of a non-critical geometrically finite one-dimensional map is Hölder continuous, while the scaling function of a critical geometrically finite one-dimensional map is discontinuous. We prove that scaling functions determine Lipschitz conjugacy classes, and moreover, that the scaling function and the exponents and asymmetries of a geometrically finite one-dimensional map are complete $C^{1}$-invariants within a mixing topological conjugacy class.
\end{abstract}

\section{InTRODUCTION}

Two smooth self-maps $f$ and $g$ of a one-dimensional compact $C^{2}$-Riemannian manifold $M$ are topologically conjugate if there is a homeomorphism $h$ from $M$ onto itself such that $h \circ f=g \circ h$. In the paper [7], we proved that if $f$ and $g$ are both geometrically finite, then $h$ is quasisymmetric [1], whence it is Hölder continuous [1]. Usually $h$ is not smooth because $f$ has a lot of smooth invariants, for example, all eigenvalues of $f$ at periodic points. Let $f$ and $g$ be orientation-preserving, expanding circle endomorphisms. Shub and Sullivan [10] proved that if $h$ is absolutely continuous, then it is smooth. Sullivan [11] also proved that if the eigenvalues of $f$ and $g$ at all corresponding periodic points are the same, then $h$ is smooth. Similar work has been done by Herman [3] for circle diffeomorphisms and by de la Llave and R. Moriyón [8] for Anosov diffeomorphisms of a torus. All these are results for maps without critical points. An important problem is to smoothly classify maps with critical points. Our first result $[4,5]$ in this direction gives the smooth classification of Ulam-von Neumann transformations (certain folding maps of an interval with one power law critical point). We proved that the eigenvalues at all periodic points together with the exponent and the asymmetry at the unique critical point of an Ulam-von Neumann transformation are complete smooth invariants. In general, all eigenvalues at the periodic points of a map may not be enough in the smooth classification of maps. To overcome this difficulty, Sullivan [12] introduced from physics (see, for example, [2]) a slightly stronger smooth invariant, the scaling function of a Cantor set, to smoothly classify certain embedded Cantor sets in the real line. In this paper, we define the scaling function for a Markov map and study the scaling function for a geometrically finite one-dimensional map. We use scaling

Received by the editors April 28, 1992 and, in revised form, March 6, 1995.

1991 Mathematics Subject Classification. Primary 58F03, 58F19, 58F34, 30F35.

The author is partially supported by PSC-CUNY awards (6-64053 and 6-65348) and an NSF grant (DMS-9400974). 
functions to smoothly classify geometrically finite one-dimensional maps within a topological conjugacy class.

Suppose $M$ is a one-dimensional compact $C^{2}$-Riemannian manifold. A geometrically finite one-dimensional map $f$ is a certain self-map of $M$ with finitely many power law singular points. We give the definition of a geometrically finite onedimensional map $f$ in $\S 3.1$. We give the definition of the dual phase space $\Sigma^{*}$ and of the scaling function $s$, on $\Sigma^{*}$, of a Markov map $f$ with a fixed Markov partition $\eta$ of $M$ to $f$ in $\S 2.2$. A singular point $c$ of a geometrically finite one-dimensional map $f$ is critical if $f$ is differentiable at $c$ and $f^{\prime}(c)=0$. A non-critical singular point is a non-differentiable point of $f$. A geometrically finite one-dimensional map $f$ is said to be critical if it has a critical singular point and to be non-critical if it has only non-critical singular points (see Definition 4 in $§ 3.1$ ). For a geometrically finite one-dimensional map $f$, there is a natural Markov partition $\eta$ of $M$ to $f$ which consists of all intervals in the complement of the orbits of singular points of $f$ in $M$. We only consider this natural Markov partition $\eta$ as a fixed Markov partition for a geometrically finite one-dimensional map $f$. Then we can say that the dual phase space $\Sigma^{*}$ of a geometrically finite one-dimensional map $f$. A function $s$ on $\Sigma^{*}$ is said to be Hölder if there are constants $C>0$ and $0<\nu<1$ such that $\left|s\left(a_{1}^{*}\right)-s\left(a_{2}^{*}\right)\right| \leq C \nu^{n}$ whenever the first $n$ digits $w_{n}$ of $a_{1}^{*}=\cdots w_{n}$ and $a_{2}^{*}=\cdots w_{n}$ in $\Sigma^{*}$ are the same. In $\S 3.2$ and $\S 3.3$ we prove the existence of the scaling function $s$ on $\Sigma^{*}$ of a geometrically finite one-dimensional map $f$.

Theorem 1. Let $f$ be a non-critical geometrically finite one-dimensional map. Then the scaling function $s$ on $\Sigma^{*}$ of $f$ exists and is Hölder.

Theorem 2. Let $f$ be a critical geometrically finite one-dimensional map. Then the scaling function $s$, on $\Sigma^{*}$, of $f$ exists.

Furthermore, because of the existence of critical points for a critical geometrically finite one-dimensional map, we prove that

Corollary 1. Let $f$ be a critical geometrically finite one-dimensional map. Then its scaling function $s$ is discontinuous on $\Sigma^{*}$.

In $\S 2.2$, we prove that the scaling function of a Markov map $f$ with a fixed Markov partition $\eta$ of $M$ to $f$ is a stronger smooth invariant than the set of all eigenvalues at periodic points of $f$ as follows: let $\Sigma^{*}$ be the dual space of $f$ with $\eta$, let $\sigma^{*}$ be the shift map of $\Sigma^{*}$, and let $h_{*}$ be a map from the set of periodic points of $\sigma^{*}$ onto the set of periodic points of $f$, which we define in $\S 2.2$.

Proposition 3. Let $f$ be a Markov map and let $\eta$ be a fixed Markov partition of $M$ to $f$. Assume that the scaling function $s$ on $\Sigma^{*}$ of $f$ with $\eta$ exists. Then for every periodic point $a^{*}$ of $\sigma^{*}$ of period $m$,

$$
\frac{1}{\left|E_{p}\right|}=\prod_{l=0}^{m-1} s\left(\left(\sigma^{*}\right)^{\circ l}\left(a^{*}\right)\right),
$$

where $p=h_{*}\left(a^{*}\right)$ is a periodic point of $f$ of period $m$ and $E_{p}=\left(f^{\circ m}\right)^{\prime}(p)$ is the eigenvalue of $f$ at $p$.

An object is said to be a $C^{1}$-invariant if it is the same for $f$ and for $h \circ f \circ h^{-1}$ whenever $h$ is a $C^{1}$-diffeomorphism of $M$. 
Proposition 4. Let $f$ be a Markov map and let $\eta$ be a fixed Markov partition of $M$ to $f$. The scaling function $s$, on $\Sigma^{*}$, of $f$ with $\eta$ (if it exists) is a $C^{1}$-invariant.

A geometrically finite one-dimensional map $f$ is said to be simple if its postcritical orbits and its set of critical points are disjoint. To avoid notational complication, we restrict discussion in $\S 3$ to simple geometrically finite one-dimensional maps. Let $\mathcal{F}$ be an (orientation-preserving) topological conjugacy class in the space of simple geometrically finite one-dimensional maps; that is, $\mathcal{F}$ is a set of simple geometrically finite one-dimensional maps such that every pair $f$ and $g$ in $\mathcal{F}$ are topologically conjugate by an orientation-preserving homeomorphism $h$ of $M$. Since all maps $f$ in $\mathcal{F}$ have the same dual phase space $\Sigma^{*}$, we may speak of the dual phase space $\Sigma^{*}$ of a topological conjugacy class $\mathcal{F}$. We note that a topological conjugacy class $\mathcal{F}$ can be described by a kneading sequence (see [9]). We prove in $\S 3.4$ that scaling functions determine Lipschitz conjugacy classes in $\mathcal{F}$ as follows:

Theorem 3. Let $f$ and $g$ be maps in $\mathcal{F}$ and let $h$ be the conjugacy from $f$ to $g$. Then $h$ is bi-Lipschitz continuous if the scaling functions $s_{f}$ and $s_{g}$, on $\Sigma^{*}$, of $f$ and $g$ are the same.

A singular point $p$ of $f$ is said to be a fold singular point if $f^{\prime}(x) f^{\prime}(2 p-x)<0$ for $x \neq p$ near $p$. Let $F S P$ be the set of all fold singular points of a geometrically finite one-dimensional map $f$, and let $F S O=\bigcup_{i=1}^{\infty} f^{\circ i}(F S P)$ be the post fold singular orbits. Let $\xi$ be the set of closures of intervals of $M \backslash F S O$. A geometrically finite one-dimensional map $f$ is mixing if for any interval $I$ in the natural Markov partition $\eta$, there is an integer $n>0$ such that $f^{\circ n}(I)=M$. Since the mixing condition is a topological invariant, we may speak of a topological conjugacy class $\mathcal{F}$ being mixing. We will prove that within a mixing topological conjugacy class $\mathcal{F}$, the scaling function $s$, on $\Sigma^{*}$, and the exponents and asymmetries of a map $f$ in $\mathcal{F}$ are complete $C^{1}$-invariants. We first prove a rigidity result for geometrically finite one-dimensional maps. Let $G S O$ be the grand singular orbits of $f$ (see §3.4).

Theorem 4. Let $f$ and $g$ be maps in a mixing topological conjugacy class $\mathcal{F}$. Let $h$ be the conjugacy from $f$ to $g$, i.e., $h \circ f=g \circ h$. Then $h \mid I$ for any $I$ in $\xi$ is a $C^{1+\beta}$-diffeomorphism for some $0<\beta \leq 1$ if and only if (i) $h$ is differentiable at one point $p$ in $M \backslash G S O$ with non-zero derivative and (ii) the exponents and asymmetries of $f$ and $g$ at all corresponding critical points are the same.

Since a Lipschitz homeomorphism $h$ is absolutely continuous, it is differentiable at almost all points with non-zero derivatives in $M$. Thus Theorems 3 and 4 tell us

Theorem 5. Let $f$ and $g$ be maps in a mixing topological conjugacy class $\mathcal{F}$ and let $\Sigma^{*}$ be the dual space of $\mathcal{F}$. Let $h$ be the topological conjugacy from $f$ to $g$, i.e., $f \circ h=h \circ g$. Then $h \mid I$ for any $I$ in $\xi$ is a $C^{1+\beta}$-diffeomorphism for some $0<\beta \leq 1$ if and only if the scaling functions $s_{f}$ and $s_{g}$, on $\Sigma^{*}$, and the exponents and asymmetries of $f$ and $g$ at corresponding critical points are the same.

\section{ACKNOWLEDGMENT}

The author would like to thank Dennis Sullivan for many insightful suggestions. 


\section{THE SCALING STRUCTURE OF A MARKOV MAP}

Let $M$ be an oriented compact one-dimensional $C^{2}$-Riemannian manifold and let $f: M \rightarrow M$ be a continuous self-map. The map $f$ is said to be Markov if there is a set $\eta=\left\{I_{1}, \cdots, I_{k}\right\}$ of closed intervals of $M$ such that

a. $I_{1}, \cdots, I_{k}$ have pairwise disjoint interiors,

b. the union $\bigcup_{i=1}^{k} I_{i}$ of all intervals in $\eta$ is $M$,

c. the restriction $f \mid I$ to every interval $I$ in $\eta$ is injective and continuous, and

d. the image $f(I)$ of every interval $I$ in $\eta$ under $f$ is the union of some intervals in $\eta$.

Remark 1. A set of closed intervals satisfying $\mathbf{a}, \mathbf{b}, \mathbf{c}$, and $\mathbf{d}$ is called a Markov partition of $M$ to $f$. For a Markov map $f$, one can find many Markov partitions of $M$ to $f$. But there is the natural one for a map which we discuss in $\S 3$.

2.1. Symbolic dynamical systems and phase spaces. Let $f$ be a Markov map and let $\eta=\left\{I_{1}, \cdots, I_{k}\right\}$ be a fixed Markov partition of $M$ to $f$. Let $g_{i}$ be the inverse of the restriction $f \mid I_{i}$ for $i=1, \cdots, k$. A sequence $w_{n}=i_{0} \cdots i_{n-1}$ of symbols $\{1, \cdots, k\}$ is said to be admissible if $I_{i_{m}} \subset f\left(I_{i_{m-1}}\right)$ for every $m=1, \cdots$, $n-1$. For an admissible sequence $w_{n}=i_{0} \cdots i_{n-1}$, define $g_{w_{n}}=g_{i_{0}} \circ \cdots \circ g_{i_{n-1}}$, and define $I_{w_{n}}=g_{w_{n}}\left(f\left(I_{i_{n-1}}\right)\right)$. We use $\eta_{n}$ to denote the set of intervals $I_{w_{n}}$ for all admissible sequences $w_{n}$ of length $n$; we call $\eta_{n}$ the $n^{\text {th }}$ partition of $M$ induced from $f$ with $\eta$. (It is also a Markov partition.) We use $\lambda_{n}$ to denote the maximum of the lengths of intervals in $\eta_{n}$. We always assume that $\lambda_{n}$ tends to zero as $n$ goes to infinity. Then $(\Sigma, \sigma)$ is the phase space of $f$ with $\eta$, where $\Sigma=\left\{a=i_{0} i_{1} \cdots\right\}$ is the set of all infinite admissible sequences with the product topology and $\sigma\left(i_{0} i_{1} \cdots\right)=i_{1} \cdots$ is the shift map of $\Sigma$.

Proposition 1. Let $f$ be a Markov map and let $\eta$ be a fixed Markov partition. Then there is a continuous map $h$ from $\Sigma$ onto $M$ such that $f \circ h=h \circ \sigma$ on $\Sigma$.

Proof. For every $a=i_{0} i_{1} \cdots$ in $\Sigma$, let $w_{n}=i_{0} i_{1} \cdots i_{n-1}$. Every $w_{n}$ is admissible and $I_{w_{n+1}} \subseteq I_{w_{n}}$ for $n>0$. Hence $\bigcap_{n=0}^{\infty} I_{w_{n}}$ is a nonempty set. But from our assumption that $\lambda_{n}$ tends to zero as $n$ goes to infinity, this set contains only one number $x_{a}$. Set $h(a)=x_{a}$. Then

$$
f(h(a))=f\left(x_{a}\right)=f\left(\bigcap_{n=0}^{\infty} I_{w_{n}}\right)=\bigcap_{n=0}^{\infty} f\left(I_{w_{n}}\right)=\bigcap_{n=0}^{\infty} I_{\sigma\left(w_{n}\right)}=h(\sigma(a)) .
$$

Now let us show that $h$ is continuous. Two points $a$ and $b$ in $\Sigma$ are $n$-close if the first $n$ digits of them are the same. Suppose $a=i_{0} \cdots i_{n-1} i_{n} \cdots$ and $b=$ $i_{0} \cdots i_{n-1} i_{n}^{\prime} \cdots$ are $n$-close. Then $h(a)$ and $h(b)$ are in the same interval $I_{w_{n}}$, where $w_{n}=i_{0} \cdots i_{n-1}$. This implies that $|h(a)-h(b)| \leq \lambda_{n}$. So $h(b)$ tends to $h(a)$ in $M$ as $b$ tends to $a$ in $\Sigma$ because $\lambda_{n}$ tends to zero as $n$ goes to infinity. This means that $h$ is continuous at every point $a$ in $\Sigma$.

The map $h$ is onto because $\bigcup_{I \in \eta_{n}} I=M$ for every $n \geq 0$. Actually, $h$ is one-to-one except for countably many points which are the preimages, under $h$, of endpoints of intervals of $\eta_{n}$, for $n \geq 0$.

The dynamical system $\sigma$ of $\Sigma$ is a topological model of a topological conjugacy class as follows: 
Proposition 2. Let $f$ be a Markov map and let $\eta$ be a fixed Markov partition of $M$ to $f$. Suppose $(\Sigma, \sigma)$ is the phase space of $f$ with $\eta$. A Markov map $g$ is topologically conjugate to $f$ if and only if there is a Markov partition $\eta^{\prime}$ of $M$ to $g$ such that the phase space of $g$ with $\eta^{\prime}$ is $(\Sigma, \sigma)$.

Proof. Suppose $g$ is topologically conjugate to $f$. There is a homeomorphism $H$ of $M$ such that $H \circ f=g \circ H$. Let $\eta^{\prime}=H(\eta)$. Then $\eta^{\prime}$ is a Markov partition of $M$ to $g$ and the phase space of $g$ with $\eta^{\prime}$ is $(\Sigma, \sigma)$.

Now suppose there is a Markov partition $\eta^{\prime}$ of $M$ to $g$ such that the phase space of $g$ with $\eta^{\prime}$ is also $(\Sigma, \sigma)$. From Proposition 1 , there are two continuous maps $h_{1}$ and $h_{2}$ from $\Sigma$ onto $M$ such that $f \circ h_{1}=h_{1} \circ \sigma$ and $g \circ h_{2}=h_{2} \circ \sigma$. Let $H=h_{2} \circ h_{1}^{-1}$. It is defined on $M$ except for countably many points which are endpoints of intervals in the partitions $\left\{\eta_{n}\right\}$ under $h_{1}$. The map $H$ is also uniformly continuous. So it can be extended to a continuous map from $M$ to $M$. Using the same argument, $H^{-1}=h_{1} \circ h_{2}^{-1}$ can be also extended to a continuous map from $M$ to $M$. Hence $H$ is a homeomorphism of $M$.

2.2. Dual symbolic spaces and scaling functions. Suppose $f$ is a Markov map and $\eta=\left\{I_{1}, \cdots, I_{k}\right\}$ is a fixed Markov partition of $M$ to $f$. In this section, we consider another symbolic space induced from $f$ with $\eta$.

Let $\Gamma_{n}$ be the set of all admissible sequences (see $\left.\S 2.1\right) w_{n}$ of length $n$. An $(n, m)$ right cylinder for $0 \leq m \leq n-1$ is $\left\{w_{n}=i_{n-1} \cdots i_{0} \in \Gamma_{n} \mid i_{l}=i_{l}^{0}, l=0, \cdots, m\right\}$, where $w_{n}^{0}=i_{n-1}^{0} \cdots i_{0}^{0}$ is a fixed sequence in $\Gamma_{n}$. All the $(n, m)$-right cylinders form a topological basis of $\Gamma_{n}$. Let $\Gamma_{n}^{*}$ be the set $\Gamma_{n}$ with this topological basis and $\left(\Sigma^{*}, \sigma^{*}\right)$ be the inverse limit of the sequence $\left\{\left(\Gamma_{n}^{*}, I_{n}^{*}\right)\right\}_{n=0}^{\infty}$, where $I_{n}^{*}: \Gamma_{n}^{*} \rightarrow \Gamma_{n-1}^{*}$ is the inclusion and $\sigma^{*}: \Sigma^{*} \rightarrow \Sigma^{*}$ is the shift. We call $\Sigma^{*}=\left\{a^{*}=\cdots i_{1} i_{0}\right\}$ the dual phase space of $f$ with $\eta$. The scaling function of $f$ with $\eta$ is a function defined on the dual phase space $\Sigma^{*}$. For $a^{*}=\cdots i_{1} i_{0}$ and $w_{n}=i_{n-1} \cdots i_{1} i_{0}$, we have $\sigma^{*}\left(a^{*}\right)=\cdots i_{1}$; we also denote $\sigma^{*}\left(w_{n}\right)$ by $i_{n-1} \cdots i_{1}$.

Let $a^{*}=\cdots w_{n}$ in $\Sigma^{*}$. Define

$$
s\left(w_{n}\right)=\frac{\left|I_{w_{n}}\right|}{\left|I_{\sigma^{*}\left(w_{n}\right)}\right|} .
$$

Definition 1. If $\lim _{n \rightarrow+\infty} s\left(w_{n}\right)$ exists for every $a^{*}$ in $\Sigma^{*}$, then we define the scaling function $s\left(a^{*}\right)=\lim _{n \rightarrow+\infty} s\left(w_{n}\right)$ on $\Sigma^{*}$.

Let $a^{*}$ be a periodic point of $\sigma^{*}$ of period $m$. Then $a^{*}=w_{m}^{\infty}=\cdots w_{m} \cdots w_{m}$ where $w_{m}=i_{m-1} \cdots i_{0}$ is an admissible sequence of length $m$. We may define $h_{*}\left(a^{*}\right)=\bigcap_{i=1}^{\infty} I_{w_{m}^{i}}$ since $I_{w_{m}^{i+1}} \subseteq I_{w_{m}^{i}} ; h_{*}\left(a^{*}\right)$ is a periodic point of $f$ of period $m$.

Proposition 3. Let $f$ be a Markov map and let $\eta$ be a fixed Markov partition of $M$ to $f$. Assume that the scaling function $s$ on $\Sigma^{*}$ of $f$ with $\eta$ exists. Then for every periodic point $a^{*}$ of $\sigma^{*}$ of period $m$,

$$
\frac{1}{\left|E_{p}\right|}=\prod_{l=0}^{m-1} s\left(\left(\sigma^{*}\right)^{\circ l}\left(a^{*}\right)\right),
$$

where $p=h_{*}\left(a^{*}\right)$ is a periodic point of $f$ of period $m$ and $E_{p}=\left(f^{\circ m}\right)^{\prime}(p)$ is the eigenvalue of $f$ at $p$. 
Proof. Suppose $a^{*}=w_{m}^{\infty}$. Then $f^{\circ m}\left(I_{w_{m}^{i+1}}\right)=I_{w_{m}^{i}}$. So

$$
\left|\left(f^{\circ m}\right)^{\prime}\left(b_{i}\right)\right|^{-1}=\frac{\left|I_{w_{m}^{i+1}}\right|}{\left|I_{w_{m}^{i}}\right|}=\prod_{l=0}^{m-1} s\left(\left(\sigma^{*}\right)^{\circ l}\left(w_{m}^{i+1}\right)\right)
$$

for some $b_{i}$ in $I_{w_{m}^{i+1}}$. As $i$ goes to infinity, $b_{i}$ tends to $p$ and $s\left(\left(\sigma^{*}\right)^{\circ l}\left(w_{m}^{i+1}\right)\right)$ tends to $s\left(\left(\sigma^{*}\right)^{\circ l}\left(a^{*}\right)\right)$. Hence

$$
\left|\left(f^{\circ m}\right)^{\prime}(p)\right|^{-1}=\prod_{l=0}^{m-1} s\left(\left(\sigma^{*}\right)^{\circ l}\left(a^{*}\right)\right) .
$$

Proposition 4. Let $f$ be a Markov map and let $\eta$ be a fixed Markov partition of $M$ to $f$. The scaling function $s$, on $\Sigma^{*}$, of $f$ with $\eta$ (if it exists) is a $C^{1}$-invariant.

Proof. Let $g=h \circ f \circ h^{-1}$ where $h$ is a $C^{1}$-diffeomorphism of $M$. Then $g$ is a Markov map and $\eta^{\prime}=h(\eta)$ is a Markov partition of $M$ to $g$. Let $s_{f}$ be the scaling function on $\Sigma^{*}$ of $f$ with $\eta$ and let $s_{g}$ be the scaling function $s$ on $\Sigma^{*}$ of $g$ with $\eta^{\prime}$.

For every $a^{*}=\cdots w_{n}, w_{n}$ is admissible and

$$
s_{g}\left(w_{n}\right)=\frac{\left|h\left(I_{w_{n}}\right)\right|}{\left|h\left(I_{\sigma^{*}}\left(w_{n}\right)\right)\right|}=\frac{\left|h^{\prime}\left(b_{n}\right)\right|}{\left|h^{\prime}\left(b_{n}^{\prime}\right)\right|} \cdot \frac{\left|I_{w_{n}}\right|}{\left.\mid I_{\sigma^{*}\left(w_{n}\right)}\right)}=\frac{\left|h^{\prime}\left(b_{n}\right)\right|}{\left|h^{\prime}\left(b_{n}^{\prime}\right)\right|} \cdot s_{f}\left(w_{n}\right)
$$

where $b_{n}$ and $b_{n}^{\prime}$ are in $I_{w_{n}}$. As $n$ goes to infinity, $s_{g}\left(w_{n}\right) \rightarrow s_{g}\left(a^{*}\right), s_{f}\left(w_{n}\right) \rightarrow$ $s_{f}\left(a^{*}\right)$, and $\left|h^{\prime}\left(b_{n}\right)\right| /\left|h^{\prime}\left(b_{n}^{\prime}\right)\right| \rightarrow 1$. Hence $s_{g}\left(a^{*}\right)=s_{f}\left(a^{*}\right)$. So $s_{f}=s_{g}$ on $\Sigma^{*}$.

\section{Geometrically finite one-Dimensional maps}

Let $M$ be a one-dimensional compact $C^{2}$-Riemannian manifold. Suppose that $f: M \rightarrow M$ is continuous and piecewise $C^{1}$. A singular point $a$ of $f$ is either a non-differentiable point or a differentiable point with zero derivative. A singular point $a$ of $f$ is said to be a power law singular point if there is a $\gamma \geq 1$ such that

$$
\lim _{x \mapsto a+} \frac{f^{\prime}(x)}{|x-a|^{\gamma-1}} \quad \text { and } \quad \lim _{x \mapsto a-} \frac{f^{\prime}(x)}{|x-a|^{\gamma-1}}
$$

exist with nonzero limits $B_{+}$and $B_{-}$. The numbers $\gamma$ and $A=B_{+} / B_{-}$are called the exponent and asymmetry of $f$ at $a$.

Remark 2. Both $\gamma$ and $A$ are orientation-preserving $C^{1}$-invariants, i.e., they are the same for $f$ and $h \circ f \circ h^{-1}$ whenever $h$ is an orientation-preserving $C^{1}$-diffeomorphism of $M$.

Henceforth, we assume that $f$ has only power law singular points and, without loss of generality, that $f$ maps the boundary of $M$ (if not empty) into itself and that the one-sided derivatives of $f$ at all boundary points of $M$ are non-zero. We note that in the general case, a boundary point of $M$ should count as a singular point anyway. We call a singular point with exponent $\gamma>1$ a critical point. Remember that a singular point with exponent $\gamma=1$ is a non-differentiable point of $f$ and that a critical point is a differentiable point of $f$ with zero derivative.

Let $N P=\left\{a_{1}, \cdots, a_{d^{\prime}}\right\}$ be the set of non-differentiable points of $f$ and let $C P=\left\{c_{1}, \cdots, c_{d}\right\}$ be the set of critical points of $f$. Let $\Gamma=\left\{\gamma_{1}, \cdots, \gamma_{d}\right\}$ be the set of corresponding exponents at critical points of $f$. Then $S=N P \cup C P$ is the set of singular points of $f$. Let $S O=\bigcup_{n=0}^{\infty} f^{\circ n}(S)$ be the set of orbits of singular points. Let $P C O=\bigcup_{n=1}^{\infty} f^{\circ n}(C P)$ be the set of post-critical orbits of $f$. 
Definition 2. The map $f$ is said to be $C^{1+\alpha}$ for some $0<\alpha \leq 1$ if

i. for every interval $I$ in the complement of $S$ in $M, f \mid I$ is $C^{1}$ and $(f \mid I)^{\prime}$ is $\alpha$-Hölder continuous, and

ii. for every critical point $c_{i}$ of $f$, there is an open neighborhood $U_{i}$ of $c_{i}$ such that $f^{\prime}(x) /\left|x-c_{i}\right|^{\gamma_{i}-1}$ is $\alpha$-Hölder continuous when restricted to $\{x<c\} \cap U_{i}$ and to $\{x>c\} \cap U_{i}$.

3.1. A geometrically finite one-dimensional map. Let $f$ be a continuous map from $M$ into $M$. If the set $S O$ of singular orbits is non-empty and finite, then $f$ is a Markov map and there is a natural Markov partition $\eta$ which consists of the closures of intervals in the complement of $S O$ in $M$. Let $\eta_{n}$ be the $n^{\text {th }}$ partition of $M$ induced from $f$ with $\eta$ and let $\lambda_{n}$ be the maximum of the lengths of intervals in $\eta_{n}$.

Definition 3. A one-dimensional map $f: M \rightarrow M$ is said to be geometrically finite if

(1) $f$ is $C^{1+\alpha}$,

(2) the set of singular orbits $S O$ is non-empty and finite,

(3) no critical point is periodic, and

(4) there are constants $C>0$ and $0<\mu<1$ such that $\lambda_{n} \leq C \mu^{n}$ for all integers $n \geq 0$.

For a geometrically finite one-dimensional map $f$, we always take the natural Markov partition $\eta$ which is the set of the closures of intervals in the complement of $S O$ in $M$, and assume that $\eta_{n}$ is the $n^{\text {th }}$ partition induced from $f$ with $\eta$ and that $\Sigma^{*}$ is the dual phase space of $f$ with $\eta$. Within this context, we can talk about the dual phase space and the scaling function of $f$. We can also fix an integer $n_{0}>0$ such that the closure $\bar{U}_{i}$ of every open interval $U_{i}$ in ii of Definition 2 is the union of two intervals in $\eta_{n_{0}}$ and such that $U=\bigcup_{i=1}^{d} U_{i}$ is disjoint with $P C O \backslash C P$ (where $P C O=\bigcup_{i=1}^{\infty} f^{\circ i}(C P)$ is the set of post-critical orbits of $f$ ). Let $V$ be the closure of the complement of $U$ in $M$. These notations will be fixed for the rest of the paper.

Definition 4. A geometrically finite one-dimensional map $f$ is said to be noncritical if it has no critical point; and $f$ is said to be critical if it has critical points.

3.2. A non-critical geometrically finite one-dimensional map. Let $f$ be a geometrically finite one-dimensional map and let $\Sigma^{*}$ be the dual phase space of $f$. A function $s$ on $\Sigma^{*}$ is said to be Hölder if there are constants $C>0$ and $0<\nu<1$ such that $\left|s\left(a_{1}^{*}\right)-s\left(a_{2}^{*}\right)\right| \leq C \nu^{n}$ whenever the first $n$ digits of $a_{1}^{*}$ and $a_{2}^{*}$ in $\Sigma^{*}$ are the same.

Theorem 1. Let $f$ be a non-critical geometrically finite one-dimensional map. Then the scaling function $s$ on $\Sigma^{*}$ of $f$ exists and is Hölder.

To prove Theorem 1, we first state and prove some important lemmas.

Lemma 1 (the naive distortion lemma). Suppose $g$ from a set $W \subseteq M$ into $M$ is a $C^{1+\alpha}$ map for some $0<\alpha \leq 1$ and suppose $a_{0}=\inf _{x \in W}\left|g^{\prime}(x)\right|>0$. Let

$b_{0}=\sup _{x \neq y \in W} \frac{\left|g^{\prime}(x)-g^{\prime}(y)\right|}{|x-y|^{\alpha}}<\infty$. Then for any two sequences $\left\{x_{i}\right\}_{i=1}^{n}$ and $\left\{y_{i}\right\}_{i=1}^{n}$ of $W$,

$$
\left|\log \left(\prod_{i=1}^{n}\left|\frac{g^{\prime}\left(x_{i}\right)}{g^{\prime}\left(y_{i}\right)}\right|\right)\right| \leq \frac{b_{0}}{a_{0}} \sum_{i=1}^{n}\left|x_{i}-y_{i}\right|^{\alpha}
$$


Proof. The proof is easy since

$$
\begin{gathered}
\left|\log \left(\prod_{i=1}^{n}\left|\frac{g^{\prime}\left(x_{i}\right)}{g^{\prime}\left(y_{i}\right)}\right|\right)\right| \leq \sum_{i=1}^{n}|\log | g^{\prime}\left(x_{i}\right)|-\log | g^{\prime}\left(y_{i}\right)|| \\
\leq \sum_{i=1}^{n} \frac{1}{a_{0}}\left|g^{\prime}\left(x_{i}\right)-g^{\prime}\left(y_{i}\right)\right| \leq \sum_{i=1}^{n} \frac{b_{0}}{a_{0}}\left|x_{i}-y_{i}\right|^{\alpha} .
\end{gathered}
$$

Lemma 2. Suppose $f$ is a non-critical geometrically finite one-dimensional map. Then there are constants $C>0$ and $0<\mu<1$ such that for any integers $n, m>0$ and any interval $I$ in $\eta_{n+m}$,

$$
\max _{x, y \in I}\left\{\left|\log \left(\frac{\left|\left(f^{\circ m}\right)^{\prime}(x)\right|}{\left|\left(f^{\circ m}\right)^{\prime}(y)\right|}\right)\right|\right\} \leq C \mu^{n} .
$$

Proof. For any $x$ and $y$ in $I$, let $x_{i}=f^{\circ i}(x)$ and $y_{i}=f^{\circ i}(y)$. From (4) of Definition 3 , there are constants $C_{1}>0$ and $0<\mu_{1}<1$ such that

$$
\left|x_{i}-y_{i}\right| \leq C_{1} \mu_{1}^{n+m-i}
$$

for $0 \leq i<m$. Let $a_{0}=\min _{x \in J \in \eta}\left|f^{\prime}(x)\right|$ and let

$$
b_{0}=\sup _{J \in \eta, x \neq y \in J} \frac{\left|f^{\prime}(x)-f^{\prime}(y)\right|}{|x-y|^{\alpha}} .
$$

Since $f$ is non-critical and $C^{1+\alpha}$, we have $0<a_{0}, b_{0}<\infty$. Applying Lemma 1 and the chain rule, there are constants $C>0$ and $0<\mu<1$ such that

$$
\left|\log \left(\frac{\left|\left(f^{\circ m}\right)^{\prime}(x)\right|}{\left|\left(f^{\circ m}\right)^{\prime}(y)\right|}\right)\right| \leq C \mu^{n}
$$

Proof of Theorem 1. Suppose $a^{*}=\cdots w_{n}$ is a point in $\Sigma^{*}$, where $w_{n}$ is the first $n$ digits of $a^{*}$ starting from the right. For any $n, m>0$, define $I_{w_{n}}=f^{\circ m}\left(I_{w_{n+m}}\right)$ and $I_{\sigma^{*}\left(w_{n}\right)}=f^{\circ m}\left(I_{\sigma^{*}\left(w_{n+m}\right)}\right)$. Hence

$$
\begin{gathered}
\left|s\left(w_{n+m}\right)-s\left(w_{n}\right)\right|=\left|\frac{\left(f^{\circ m}\right)^{\prime}(x)}{\left(f^{\circ m}\right)^{\prime}(y)}-1\right| \cdot s\left(w_{n}\right) \\
=\left|\frac{\left(f^{\circ m}\right)^{\prime}(x)}{\left(f^{\circ m}\right)^{\prime}(y)}-1\right| \cdot\left|\frac{\left(f^{\circ n}\right)^{\prime}\left(x^{\prime}\right)}{\left(f^{\circ n}\right)^{\prime}\left(y^{\prime}\right)}\right| \cdot s\left(w_{1}\right)
\end{gathered}
$$

where $x$ and $y$ are in $I_{w_{n+m}}$ and $x^{\prime}$ and $y^{\prime}$ are in $I_{w_{n}}$. From Lemma 2, there are constants $C>0$ and $0<\mu<1$ such that

$$
\left|\frac{\left(f^{\circ m}\right)^{\prime}(x)}{\left(f^{\circ m}\right)^{\prime}(y)}-1\right| \leq C \mu^{n} \quad \text { and } \quad\left|\frac{\left(f^{\circ n}\right)^{\prime}\left(x^{\prime}\right)}{\left(f^{\circ n}\right)^{\prime}\left(y^{\prime}\right)}\right| \cdot s\left(w_{1}\right) \leq C .
$$

Thus $\left|s\left(w_{n+m}\right)-s\left(w_{n}\right)\right| \leq C^{2} \mu^{n}$ for all $m>0$, whence $\left\{s\left(w_{n}\right)\right\}_{n=0}^{\infty}$ is a Cauchy sequence. This implies that the scaling function $s\left(a^{*}\right)=\lim _{n \rightarrow \infty} s\left(w_{n}\right)$ exists.

To prove that the scaling function $s$ on $\Sigma^{*}$ is Hölder, we consider two points $a^{*}=\cdots w_{n+m}$ and $b^{*}=\cdots w_{n+m}^{\prime}$ whose first $n$ digits are the same; that is, $w_{n}=$ $w_{n}^{\prime}$. Following the previous argument, 


$$
\begin{gathered}
\left|s\left(w_{n+m}\right)-s\left(w_{n+m}^{\prime}\right)\right|=\left|\frac{\left(f^{\circ m}\right)^{\prime}(x)}{\left(f^{\circ m}\right)^{\prime}(y)}-\frac{\left(f^{\circ m}\right)^{\prime}\left(x^{\prime}\right)}{\left(f^{\circ m}\right)^{\prime}\left(y^{\prime}\right)}\right| \cdot s\left(w_{n}\right) \\
=\left|\frac{\left(f^{\circ m}\right)^{\prime}(x)}{\left(f^{\circ m}\right)^{\prime}(y)}-\frac{\left(f^{\circ m}\right)^{\prime}\left(x^{\prime}\right)}{\left(f^{\circ m}\right)^{\prime}\left(y^{\prime}\right)}\right| \cdot\left|\frac{\left(f^{\circ n}\right)^{\prime}\left(x^{\prime \prime}\right)}{\left(f^{\circ n}\right)^{\prime}\left(y^{\prime \prime}\right)}\right| \cdot s\left(w_{1}\right)
\end{gathered}
$$

where $x$ and $y$ are in $I_{w_{n+m}}, x^{\prime}$ and $y^{\prime}$ are in $I_{w_{n+m}^{\prime}}$, and $x^{\prime \prime}$ and $y^{\prime \prime}$ are in $I_{w_{n}}$. Hence

$$
\left|s\left(w_{n+m}\right)-s\left(w_{n+m}^{\prime}\right)\right| \leq 2 C^{2} \mu^{n} .
$$

As $m$ goes to infinity, we have

$$
\left|s\left(a^{*}\right)-s\left(b^{*}\right)\right| \leq 2 C^{2} \mu^{n}
$$

that is, $s$ is Hölder continuous.

3.3. A critical geometrically finite one-dimensional map. Suppose $f$ is a critical geometrically finite one-dimensional map. Let $C P=\left\{c_{1}, \cdots, c_{d}\right\}$ be the set of critical points of $f$ and let $\Gamma=\left\{\gamma_{1}, \cdots, \gamma_{d}\right\}$ be the set of corresponding exponents.

Theorem 2. Let $f$ be a critical geometrically finite one-dimensional map. Then the scaling function $s$, on $\Sigma^{*}$, of $f$ exists.

Furthermore, because of the existence of critical points for a critical geometrically finite one-dimensional map, we have

Corollary 1. Let $f$ be a critical geometrically finite one-dimensional map. Then its scaling function $s$ is discontinuous on $\Sigma^{*}$.

We note that since $\inf _{I \in \eta, x \in I}\left|f^{\prime}(x)\right|=0$ for a critical geometrically finite onedimensional map, we can not applying Lemma 1 directly. To overcome this difficulty, we first prove two distortion lemmas. The integer $n_{0}$ and the sets $U$ and $V$ are the same as in $\S 3.1$. For a point $x$ in $M$, let $x_{i}=f^{\circ i}(x)$.

Lemma 3. There are constants $C>0$ and $0<\mu<1$ such that for any integers $n \geq n_{0}$ and $m>0$, and for any points $x$ and $y$ in an interval of $\eta_{n+m}$, if $x_{i}$ and $y_{i}$ are in $V$ for all $0 \leq i<m$, then

$$
\left|\log \left(\frac{\left|\left(f^{\circ m}\right)^{\prime}(x)\right|}{\left|\left(f^{\circ m}\right)^{\prime}(y)\right|}\right)\right| \leq C \mu^{n} .
$$

Proof. Suppose $f$ is $C^{1+\alpha}$. Let $a_{0}=\min _{x \in V}\left|f^{\prime}(x)\right|$ and

$$
b_{0}=\sup _{x \neq y \in V} \frac{\left|f^{\prime}(x)-f^{\prime}(y)\right|}{|x-y|^{\alpha}} .
$$

Then $0<a_{0}, b_{0}<\infty$. From Lemma 1 ,

$$
\left|\log \left(\frac{\left|\left(f^{\circ m}\right)^{\prime}(x)\right|}{\left|\left(f^{\circ m}\right)^{\prime}(y)\right|}\right)\right| \leq \frac{b_{0}}{a_{0}} \sum_{i=0}^{m-1}\left|x_{i}-y_{i}\right|^{\alpha} .
$$

Since $x$ and $y$ are in an interval in $\eta_{n+m}, x_{i}$ and $y_{i}$ are in an interval in $\eta_{n+m-i}$. Hence $\left|x_{i}-y_{i}\right| \leq C \mu^{n+m-i}$ because of (4) of Definition 3. Hence there are constants $C>0$ and $0<\mu<1$ such that

$$
\left|\log \left(\frac{\left|\left(f^{\circ m}\right)^{\prime}(x)\right|}{\left|\left(f^{\circ m}\right)^{\prime}(y)\right|}\right)\right| \leq C \mu^{n} .
$$


The next lemma is one of the key lemmas in this paper. To present a clear idea of the proof, we first prove it under the assumption that $P C O \cap C P=\emptyset$. For a point $x$ in $M$, let $x_{i}=f^{\circ i}(x)$.

Lemma 4. There are constants $C>0$ and $0<\mu<1$ such that for any integers $n \geq n_{0}$ and $m>0$ and any points $x$ and $y$ in an interval of $\eta_{n+m}$, if $x_{m}$ and $y_{m}$ are in $U$, then

$$
\left|\log \left(\frac{\left|\left(f^{\circ m}\right)^{\prime}(x)\right|}{\left|\left(f^{\circ m}\right)^{\prime}(y)\right|}\right)\right| \leq C \mu^{n} .
$$

Proof. The ratio $\left|\left(f^{\circ m}\right)^{\prime}(x)\right| /\left|\left(f^{\circ m}\right)^{\prime}(y)\right|$ equals the product $\prod_{i=0}^{m-1}\left|f^{\prime}\left(x_{i}\right)\right| /\left|f^{\prime}\left(y_{i}\right)\right|$. We divide this product into two sub-products,

$$
\prod_{x_{i}, y_{i} \in U} \frac{\left|f^{\prime}\left(x_{i}\right)\right|}{\left|f^{\prime}\left(y_{i}\right)\right|} \text { and } \prod_{x_{i}, y_{i} \in V} \frac{\left|f^{\prime}\left(x_{i}\right)\right|}{\left|f^{\prime}\left(y_{i}\right)\right|} .
$$

Following the proof of Lemma 3 , there are constants $C_{1}, C_{1}^{\prime}>0$ and $0<\mu_{1}<1$ such that

$$
\left|\log \left(\prod_{x_{i}, y_{i} \in V} \frac{\left|f^{\prime}\left(x_{i}\right)\right|}{\left|f^{\prime}\left(y_{i}\right)\right|}\right)\right| \leq C_{1} \sum_{x_{i}, y_{i} \in V}\left|x_{i}-y_{i}\right|^{\alpha} \leq C_{1}^{\prime} \mu_{1}^{n} .
$$

To estimate the first product $\prod_{x_{i}, y_{i} \in U}\left|f^{\prime}\left(x_{i}\right)\right| /\left|f^{\prime}\left(y_{i}\right)\right|$, we write it as the product of three factors:

$$
\begin{gathered}
\mathcal{I}=\prod_{x_{i}, y_{i} \in U}\left(\frac{\left|x_{i}-c_{k_{i}}\right|^{\gamma_{k_{i}}}}{\left|f\left(x_{i}\right)-f\left(c_{k_{i}}\right)\right|} \frac{\left|f\left(y_{i}\right)-f\left(c_{k_{i}}\right)\right|}{\left|y_{i}-c_{k_{i}}\right|^{\gamma_{k_{i}}}}\right)^{t_{k_{i}}}, \\
\mathcal{I} \mathcal{I}=\prod_{x_{i}, y_{i} \in U} \frac{\left|y_{i}-c_{k_{i}}\right|^{\gamma_{k_{i}}-1}}{\left|f^{\prime}\left(y_{i}\right)\right|} \frac{\left|f^{\prime}\left(x_{i}\right)\right|}{\left|x_{i}-c_{k_{i}}\right|^{\gamma_{k_{i}}-1}},
\end{gathered}
$$

and

$$
\mathcal{I I I}=\prod_{x_{i}, y_{i} \in U}\left(\frac{\left|f\left(x_{i}\right)-f\left(c_{k_{i}}\right)\right|}{\left|f\left(y_{i}\right)-f\left(c_{k_{i}}\right)\right|}\right)^{t_{k_{i}}}
$$

where $x_{i}$ and $y_{i}$ are in $U_{k_{i}}$ and $t_{k_{i}}=\left(\gamma_{k_{i}}-1\right) / \gamma_{k_{i}}$. Applying Lemma 1 and (1) of Definition 3 , and following the proof of Lemma 1 , there are constants $C_{2}, C_{2}^{\prime}>0$ and $0<\mu_{2}<1$ such that

$$
|\log \mathcal{I}|,|\log \mathcal{I I}| \leq C_{2} \sum_{x_{i}, y_{i} \in U}\left|x_{i}-y_{i}\right|^{\alpha} \leq C_{2}^{\prime} \mu_{2}^{n}
$$

Now the proof of Lemma 4 concentrates on the estimate of $\mathcal{I} \mathcal{I} \mathcal{I}$. Let

$$
\frac{f\left(x_{i}\right)-f\left(c_{k_{i}}\right)}{f\left(y_{i}\right)-f\left(c_{k_{i}}\right)}=1+\frac{f\left(x_{i}\right)-f\left(y_{i}\right)}{f\left(y_{i}\right)-f\left(c_{k_{i}}\right)} .
$$

Then

$$
\mathcal{I I I}=\exp \left(\sum_{s=1}^{r-1} \frac{1}{t_{k_{s}}} \log \left|1+\frac{\left|f\left(x_{i_{s}}\right)-f\left(y_{i_{s}}\right)\right|}{\mid f\left(y_{i_{s}}\right)-f\left(c_{k_{i_{s}}}\right)}\right|\right)
$$

where $i_{1}<i_{2}<\cdots<i_{r-1}<m$. Let $i_{r}=m$. For each $i_{s}, 1 \leq s<r$, consider the interval $L_{s}$ bounded by $y_{i_{s}}$ and $c_{k_{i_{s}}}$ and the map $h_{s}=f^{\circ\left(i_{s+1}-i_{s}\right)}$. Let $R_{s} \subseteq L_{s}$ be the maximal interval containing $y_{i_{s}}$ such that $h_{s}$ on $R_{s}$ is injective. One of the endpoints of $R_{s}$ is $y_{i_{s}}$ and the other is a preimage $e$ of a critical point $c_{j_{s}}$ in $C P$ under $f^{\circ k_{s}}$ for some $0 \leq k_{s}<i_{s+1}-i_{s}$. Let $l_{s}=i_{s+1}-i_{s}-k_{s}$. Then $h_{s}$ on the minimal interval $J_{s}$ containing $x_{i_{s}}$ and $R_{s}$ is injective and maps $J_{s}$ onto an interval 
containing the points $y_{i_{s+1}}, x_{i_{s+1}}$ and $f^{\circ l_{s}}\left(c_{j_{s}}\right)$. We enlarge every interval $J$ of $V$ into a closed interval $J^{\prime} \supset J$ such that $J^{\prime} \cap C P=\emptyset$ and such that the length of $J^{\prime} \cap U$ is greater than a constant $a>0$. Let $V^{\prime}=\bigcup_{J \in V} J^{\prime}$ be the union of all these enlarged intervals and let $U^{\prime}=M \backslash V^{\prime}$. If $f^{\circ i}\left(J_{s}\right) \subseteq V^{\prime}$ for all $1 \leq i<i_{s+1}-i_{s}$, by following the proof of Lemma 3 , there is a constant $C_{3}>0$ such that

$$
\frac{\left|f\left(x_{i_{s}}\right)-f\left(y_{i_{s}}\right)\right|}{\left|f\left(y_{i_{s}}\right)-f\left(c_{k_{i_{s}}}\right)\right|} \leq C_{3} \frac{\left|x_{i_{s+1}}-y_{i_{s+1}}\right|}{\left|y_{i_{s+1}}-f^{\circ l_{s}}\left(c_{j_{s}}\right)\right|} .
$$

Since $y_{i_{s+1}}$ is in $U$ and $f^{\circ l_{s}}\left(c_{j_{s}}\right)$ is in $P C O$,

$$
\frac{\left|f\left(x_{i_{s}}\right)-f\left(y_{i_{s}}\right)\right|}{\left|f\left(y_{i_{s}}\right)-f\left(c_{k_{i_{s}}}\right)\right|} \leq C_{3} \frac{\left|x_{i_{s+1}}-y_{i_{s+1}}\right|}{D}
$$

where $D>0$ is the distance between $U$ and the post-critical orbits $P C O$. Otherwise, let $0<k<i_{s+1}-i_{s}$ be the smallest integer such that $f^{\circ k}\left(J_{s}\right) \cap U^{\prime} \neq \emptyset$. Since $f^{\circ i}\left(J_{s}\right) \subseteq V^{\prime}$ for all $1 \leq i<k$, following the proof of Lemma 3 , there is a constant $C_{4}>0$ such that

$$
\frac{\left|f\left(x_{i_{s}}\right)-f\left(y_{i_{s}}\right)\right|}{\left|f\left(y_{i_{s}}\right)-f\left(c_{k_{i_{s}}}\right)\right|} \leq C_{4} \frac{\left|x_{i_{s}+k}-y_{i_{s}+k}\right|}{\left|y_{i_{s}+k}-f^{\circ k}(e)\right|} .
$$

Since $y_{i_{s}+k}$ is in $V$ and $f^{\circ k}(e)$ is in $U^{\prime}$,

$$
\frac{\left|f\left(x_{i_{s}}\right)-f\left(y_{i_{s}}\right)\right|}{\left|f\left(y_{i_{s}}\right)-f\left(c_{k_{i_{s}}}\right)\right|} \leq C_{4} \frac{\left|x_{i_{s}+k}-y_{i_{s}+k}\right|}{D^{\prime}}
$$

where $D^{\prime}>0$ is the distance between $V$ and $U^{\prime}$. Hence, there are constants $C_{5}>0$, $C_{5}^{\prime}>0$ and $0<\mu_{4}<1$ such that

$$
|\log \mathcal{I} \mathcal{I} \mathcal{I}| \leq C_{5} \sum_{i=0}^{m-1}\left|x_{i}-y_{i}\right| \leq C_{5}^{\prime} \mu_{4}^{n} .
$$

Combining all the estimates, we have constants $C>0$ and $0<\mu<1$ satisfying the lemma.

If $P C O \cap C P \neq \emptyset$, we need to consider a critical chain: a subset $\left\{c_{i_{1}}, \cdots, c_{i_{l}}\right\}$ of $C P$ is a critical chain if $f^{\circ j_{k}}\left(c_{i_{k}}\right)=c_{i_{k+1}}$ for some integers $j_{k}>0$ and $\left\{f^{\circ j}\left(c_{i_{k}}\right)\right\}_{j=1}^{j_{k}-1}$ $\cap C P=\emptyset$.

Let $x$ and $y$ be two points in an interval in $\eta_{n+m}$, where $n \geq n_{0}$ and $m>0$. Let $x_{i}=f^{\circ i}(x)$ and $y_{i}=f^{\circ i}(y)$ for $0 \leq i \leq m$. Suppose $x_{m}$ and $y_{m}$ are in $U$. Using the same notation as in the proof of Lemma 4 , we consider

$$
\mathcal{I I I}=\exp \left(\sum_{s=1}^{r-1} \frac{1}{t_{k_{s}}} \log \left|1+\frac{\left|f\left(x_{i_{s}}\right)-f\left(y_{i_{s}}\right)\right|}{\left|f\left(y_{i_{s}}\right)-f\left(c_{k_{i_{s}}}\right)\right|}\right|\right)
$$

where $i_{1}<i_{2}<\cdots<i_{r-1}<m$ and $x_{i_{s}}$ and $y_{i_{s}}$ are in $U_{k_{i_{s}}}$. (Note that $c_{k_{i_{s}}} \in U_{k_{i_{s}}}$.) Let $i_{r}=m$ and denote $c(s)=c_{k_{i_{s}}}$ for $1 \leq s \leq r$. We divide $\{c(s)\}_{s=1}^{m}$ into maximal critical chains $\mathcal{I}_{1}=\left\{c(1), \cdots, c\left(s_{1}\right)\right\}, \mathcal{I}_{2}=\left\{c\left(s_{1}+1\right), \cdots, c\left(s_{2}\right)\right\}, \cdots$, $\mathcal{I}_{q}=\left\{c\left(s_{q-1}+1\right), \cdots, c\left(s_{q}\right)\right\}$. Remember that $c\left(s_{q}\right)=c_{k_{m}}$. The number of points in every maximal critical chain is less than or equal to the number of points in $C P$. Now we can generalize Lemma 4.

Lemma $4^{\prime}$. There are constants $C>0$ and $0<\mu<1$ such that for any integers $n \geq n_{0}$ and $m>0$ and for any points $x$ and $y$ in an interval of $\eta_{n+m}$, if $x_{m}$ and 
$y_{m}$ are in $U$ and if the last critical chain $\mathcal{I}_{q}$ contains only one critical point $c_{k_{m}}$, then

$$
\left|\log \left(\frac{\left|\left(f^{\circ m}\right)^{\prime}(x)\right|}{\left|\left(f^{\circ}\right)^{\prime}(y)\right|}\right)\right| \leq C \mu^{n}
$$

Proof. We use the same notation as in the proof of Lemma 4. The estimates of $\mathcal{I}$ and $\mathcal{I} \mathcal{I}$ are the same as in that proof. Here we add maximal critical chains in the estimates of $\mathcal{I} \mathcal{I}$. Consider a critical point $c(l)$ in a maximal critical chain $\mathcal{I}_{i}=\left\{c\left(s_{j-1}+1\right), \cdots, c\left(s_{j}\right)\right\}$ for $1 \leq j<q$. By arguments similar to those in the proof of Lemma 4 and by the fact that $f$ is comparable to $B_{ \pm}|x-c(l)|^{\gamma(l)}+f(c(l))$ near $c(l)$, there is a positive constant $C_{6}$ such that

$$
\frac{\left|f\left(x_{i_{l}}\right)-f\left(y_{i_{l}}\right)\right|}{\left|f\left(y_{i_{l}}\right)-f\left(c_{k_{i_{l}}}\right)\right|} \leq C_{6} \frac{\left|x_{i_{s_{j}+1}}-y_{i_{s_{j}+1}}\right|^{\frac{1}{\tau_{j}}}}{D^{\prime \prime}}
$$

where $\tau_{j}=\prod_{l=s_{j-1}+1}^{s_{j}} \gamma(l) \leq \gamma=\prod_{i=1}^{d} \gamma_{i}$ and where $D^{\prime \prime}$ is a constant. Therefore, there are constants $C_{5}>0$ and $0<\mu_{5}<1$ such that

$$
|\log \mathcal{I} I I| \leq C_{5} \mu_{5}^{n}
$$

Combining the estimates for $\mathcal{I}$ and $\mathcal{I} \mathcal{I}$ together, there are constants $C>0$ and $0<\mu<1$ satisfying the lemma.

Proof of Theorem 2. Suppose $a^{*}=\ldots w_{n}$ is a point in $\Sigma^{*}$ and $v_{n}=\sigma^{*}\left(w_{n}\right)$. Then $w_{n}=v_{n} i_{0}$. We discuss the sequence $\left\{I_{v_{n}}\right\}_{n=1}^{\infty}$ in two cases: (1) there is an integer $n_{1} \geq n_{0}$ such that $n \geq n_{1}$ implies $I_{v_{n}} \subset V$, (2) there is an increasing subsequence $\left\{n_{k}\right\}_{k=1}^{\infty}$ of integers such that $I_{v_{n_{k}}} \subseteq U$ for all $n_{k}$.

In (1), by arguments similar to those in the proof of Theorem 1, we can prove that $\left\{s\left(w_{n}\right)\right\}_{n=1}^{\infty}$ is a Cauchy sequence. Hence the limit $s\left(a^{*}\right)=\lim _{n \mapsto \infty} s\left(w_{n}\right)$ exists.

In (2), consider all $I_{m_{k}} \subseteq U_{m_{k}} \subset U$ and $\left\{c_{m_{k}}\right\}_{k=1}^{\infty}$. We can find an increasing sequence $\left\{m_{k_{i}}\right\}_{i=1}^{\infty}$ such that the last critical chain of $\left\{c_{m_{k}}\right\}_{k=k_{i}}^{\infty}$ contains only one critical point for every $1 \leq i<\infty$. Let $n_{i}=m_{k_{i}}$. For any $n \geq n_{i}$, the intervals $I_{w_{n_{i}}}$ and $I_{v_{n_{i}}}$ are the images of $I_{w_{n}}$ and $I_{v_{n}}$ under $f^{\circ\left(n-n_{i}\right)}$, and the intervals $I_{w_{n_{1}}}$ and $I_{v_{n_{1}}}$ are the images of $I_{w_{n_{i}}}$ and $I_{v_{n_{i}}}$ under $f^{\circ\left(n_{i}-n_{1}\right)}$. Thus

$$
\begin{aligned}
& \left|s\left(w_{n}\right)-s\left(w_{n_{i}}\right)\right|=\left|\frac{\left(f^{\circ}\left(n-n_{i}\right)\right)^{\prime}(x)}{\left(f^{\circ\left(n-n_{i}\right)}\right)^{\prime}(y)}-1\right| \cdot \frac{\left|I_{w_{n_{i}}}\right|}{\left|I_{v_{n_{i}}}\right|}
\end{aligned}
$$

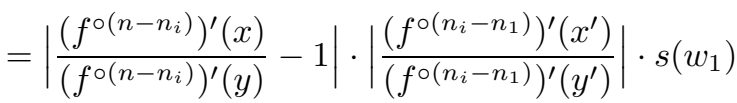

where $x$ and $y$ are in $I_{v_{n}}$ and $x^{\prime}$ and $y^{\prime}$ are in $I_{v_{n_{i}}}$. By Lemma $4^{\prime}$, there are constants $C_{1}>0$ and $0<\mu_{1}<1$ such that

$$
\left|\frac{\left(f^{\circ\left(n-n_{i}\right)}\right)^{\prime}(x)}{\left(f^{\circ\left(n-n_{i}\right)}\right)^{\prime}(y)}-1\right| \leq C \mu^{n_{i}} \quad \text { and } \quad\left|\frac{\left(f^{\circ\left(n_{i}-n_{1}\right)}\right)^{\prime}\left(x^{\prime}\right)}{\left(f^{\circ\left(n_{i}-n_{1}\right)}\right)^{\prime}\left(y^{\prime}\right)}\right| \cdot s\left(w_{1}\right) \leq C
$$

for $n \geq n_{i}$. Thus

$$
\left|s\left(w_{n}\right)-s\left(w_{n_{i}}\right)\right| \leq C^{2} \mu^{n_{i}}
$$

for $n \geq n_{i}$. The sequence $\left\{s\left(w_{n}\right)\right\}_{n=1}^{\infty}$ is thus a Cauchy sequence and $\lim _{n \rightarrow \infty} s\left(w_{n}\right)$ $=s\left(a^{*}\right)$ exists. 
Proof of Corollary 1. Suppose $c$ is a critical point of $f$. It is not periodic and its orbit $\left\{f^{\circ n}(c)\right\}_{n=0}^{\infty}$ is finite. There is a periodic point $p \neq c$ and an integer $l>0$ such that $f^{\circ i}(c) \neq p$ for $0 \leq i<l$ and $f^{\circ l}(c)=p$. Let $a^{*}=w_{m}^{\infty}$ be a point in $\Sigma^{*}$ such that $\{p\}=\bigcap_{i=1}^{\infty} I_{w_{m}^{i}}$ where $m$ is the period of $p$. From (4) of Definition 3 and from Proposition 3, we have $\left|E_{p}\right|=\left|\left(f^{\circ m}\right)^{\prime}(p)\right|>1$ and the existence of an integer $0 \leq m_{0}<m$ such that for $c^{*}=\left(\sigma^{*}\right)^{\circ m_{0}}\left(a^{*}\right), 0<s\left(c^{*}\right)<1$. We will prove that $s$ is discontinuous at $c^{*}$.

Suppose $c^{*}=u_{m}^{\infty}$. Without loss of generality, we assume that $\left\{f^{\circ i}(c)\right\}_{i=1}^{m-1}$ contains no critical points and that $c$ is not the image of any critical point under any iterate of $f$. For any $u_{m}^{i}$, there is a $b^{*}=\cdots v_{n} u_{m}^{i}$ in $\Sigma^{*}$ such that $I=I_{v_{n} u_{m}^{i}}$ contains $c$. For large $i>0, I$ is contained in $U$ and $f^{\circ j}(I)$ is contained in $V$ for every $0<j \leq n$. Since $f \mid I$ is asymptotically $B_{ \pm}|x-c|^{\gamma}+f(c)$ as $n$ tends to infinity where $\gamma>1$, Lemma 3 implies that,

$$
\lim _{i \rightarrow \infty} s\left(v_{n} u_{m}^{i}\right)=\lim _{i \mapsto \infty}\left(s\left(u_{m}^{i}\right)\right)^{\gamma}=\left(s\left(c^{*}\right)\right)^{\gamma} .
$$

From Lemma $4^{\prime}$ and from the proof of Theorem 2, $\left|s\left(b^{*}\right)-s\left(v_{n} w_{m}^{i}\right)\right| \leq C \mu^{n+m^{i}}$. We have

$$
\lim _{b^{*} \rightarrow c^{*}} s\left(b^{*}\right)=\left(s\left(c^{*}\right)\right)^{\gamma} .
$$

Hence $c^{*}$ is a jump discontinuity of $s$.

Remark 3. From the proof of Corollary 1, we can actually find out all continuous points and discontinuous points of the scaling function of a critical geometrically finite one-dimensional map. A point $a^{*}=\cdots w_{n}$ in $\Sigma_{f}^{*}$ is said to be recurrent if there is an increasing subsequence $\left\{n_{i}\right\}_{i=1}^{\infty}$ of integers such that $I_{w_{n_{i}}} \subseteq U$ for every $n_{i}$. It is said to be non-recurrent if there is an integer $n>0$ such that all preimages of $I_{w_{n}}$ under iterates of $f$ are contained in $V$. The point $a^{*}$ is said to be wandering if there is an integer $m>0$ such that $I_{w_{n}} \subseteq V$ for all $n \geq m$, and if for every integer $n \geq m$, there is another integer $\bar{n}$ such that there is an interval in the preimage of $I_{w_{n}}$ under $f^{\circ \bar{n}}$ contained in $U$. We can prove that $s$ on $\Sigma^{*}$ is continuous at all recurrent $a^{*}$, all non-recurrent $a^{*}$, and all wandering points $a^{*}$ with $s\left(a^{*}\right)=1$, and that $s$ is discontinuous at wandering points $a^{*}$ with $0<s\left(a^{*}\right)<1$. All discontinuities are jump discontinuities.

Remark 4. From the proof of Corollary 1, if $P C O \cap C P=\emptyset$, then the exponent $\gamma>1$ of $f$ at a critical point $c$ can be calculated from the scaling function $s$. Hence the exponent $\gamma$ is a $C^{1}$-invariant, by Proposition 4 .

3.4. Complete smooth invariants. A geometrically finite one-dimensional map $f$ is simple if $P C O \cap C P=\emptyset$. To avoid notational complication, we consider only simple geometrically finite one-dimensional maps in this section. Let $\mathcal{F}$ be an (orientation-preserving) topological conjugacy class in the space of simple geometrically finite one-dimensional maps, i.e., $\mathcal{F}$ is a subset of simple geometrically finite one-dimensional maps such that every pair of maps $f$ and $g$ in $\mathcal{F}$ are topologically conjugate by an orientation-preserving homeomorphism $h$. Since all maps $f$ in $\mathcal{F}$ have the same dual phase space $\Sigma^{*}$ we may speak of the dual phase space $\Sigma^{*}$ of a topological conjugacy class $\mathcal{F}$.

Remark 5. Take a fixed orientation-reversing homeomorphism $h_{0}$ of $M$. If $f$ and $g$ are topologically conjugate by an orientation-reversing homeomorphism $h$, then 
$f$ and $\tilde{g}=h_{0} \circ g \circ h_{0}^{-1}$ are topologically conjugate by an orientation-preserving homeomorphism.

Suppose $f$ and $g$ are in $\mathcal{F}$ and $h$ is the conjugacy from $f$ to $g$, i.e., $h \circ f=g \circ h$. In the paper [7], we proved that $h$ must be a quasisymmetric homeomorphism [1]. This implies that $h$ is $\alpha$-Hölder continuous for some $0<\alpha \leq 1$ [1]. Usually, $h$ is not Lipschitz because $f$ has a lot of Lipschitz invariants, for example, all eigenvalues of $f$ at periodic points. However, we have

Theorem 3. Let $f$ and $g$ be maps in $\mathcal{F}$ and let $h$ is the conjugacy from $f$ to $g$. Then $h$ is bi-Lipschitz continuous if the scaling functions $s_{f}$ and $s_{g}$ on $\Sigma^{*}$ of $f$ and $g$ are the same.

Proof. Let $\eta$ be the natural Markov partition of $M$ to $f$. Let $\eta_{n}$ be the $n^{\text {th }}$ partition induced by $f$ with $\eta$. Let $m_{0}$ be the number of the intervals in $\eta$ and define $k_{0}=m_{0}+n_{0}$, where $n_{0}$ is the fixed integer in $\S 3.1$. For any integer $n>0$ and any interval $I_{w_{k_{0}} w_{n}}$ in $\eta_{n+k_{0}}$,

$$
\frac{\left|h\left(I_{w_{k_{0}} w_{n}}\right)\right|}{\left|I_{w_{k_{0}} w_{n}}\right|}=\frac{\left|s_{g}\left(w_{k_{0}} w_{n}\right)\right|}{\left|s_{f}\left(w_{k_{0}} w_{n}\right)\right|} \cdot \frac{\left|h\left(I_{w_{k_{0}} v_{n}}\right)\right|}{\left|I_{w_{k_{0}} v_{n}}\right|}
$$

where $w_{k_{0}} v_{n}=\sigma^{*}\left(w_{k_{0}} w_{n}\right)$.

Let $a^{*}=\cdots u_{m} w_{k_{0}} w_{n}$ be a point in $\Sigma^{*}$. For the sequence $\left\{I_{u_{m} w_{k_{0}} w_{n}}\right\}_{m=0}^{\infty}$ we consider three cases: (1) $I_{w_{k_{0}} w_{n}}$ is contained in $U$; (2) $I_{u_{m} w_{k_{0}} w_{n}}$ is contained in $V$ for every $m \geq 0 ;(3)$ there is an integer $m \geq 1$ such that $I_{u_{i} w_{k_{0}} w_{n}}$ is contained in $V$ for every $0 \leq i \leq m$ and $I_{u_{m+1} w_{k_{0} w_{n}}}$ is contained in $U$.

In case (1), we use Lemma 4 and Lemma 3 for both $f$ and $g$ to find constants $C_{1}>0$ and $0<\mu_{1}<1$ such that

and

$$
\left|\log \left(\frac{s_{f}\left(a^{*}\right)}{s_{f}\left(w_{k_{0}} w_{n}\right)}\right)\right| \leq C_{1} \mu_{1}^{n}
$$

$$
\left|\log \left(\frac{s_{g}\left(a^{*}\right)}{s_{g}\left(w_{k_{0}} w_{n}\right)}\right)\right| \leq C_{1} \mu_{1}^{n} .
$$

Because $s_{g}=s_{f}$, for $C_{2}=2 C_{1}$ and $\mu_{2}=\mu_{1}$,

$$
\left|\log \left(\frac{s_{f}\left(w_{k_{0}} w_{n}\right)}{s_{g}\left(w_{k_{0}} w_{n}\right)}\right)\right| \leq C_{2} \mu_{2}^{n} .
$$

In case (3), suppose $I$ is the interval in $\eta_{k_{0}}$ having a critical point $c$ of $f$ as an endpoint and containing $I_{u_{m+1} w_{k_{0}} w_{n}}$. Then there is an integer $0<l<m_{0}$ such that $f^{\circ l}(c)$ is a periodic point $p$ of $f$ and $f^{\circ l}\left(I_{u_{m+1} w_{k_{0}} w_{n}}\right) \subset f^{\circ l}(I)$. We note that $f^{\circ l}(I)$ is an interval in $\eta_{k_{0}-l}$ and that $f^{\circ l}\left(I_{u_{m+1} w_{k_{0}} w_{n}}\right)$ is an interval in $\eta_{m+1+k_{0}+n-l}$. Remember that $p$ is an endpoint of $f^{\circ l}(I)$. We can now find a point $b^{*}=\cdots w_{j}^{\prime}$ in $\Sigma^{*}$ such that the first $m+1+k_{0}+n-l$ digits of $a^{*}$ and $b^{*}$ (starting from the right) are the same and such that the interval $I_{w_{j}^{\prime}}$ in $\eta_{j}$ is contained in $V$ for every $j>0$ and tends to the periodic orbit $\bigcup_{i=0}^{\infty} f^{\circ i}(p)$ as $j$ goes to infinity. Thus we get another sequence $\left\{I_{w_{j}^{\prime}}\right\}$ which is in case (2). If $m+1-l>0$, then $I_{w_{k_{0}} w_{n}}$ is in $\left\{I_{w_{j}^{\prime}}\right\}$. If $m+1-l<0$, then we have that $I_{w_{k_{0}-l} w_{n}}$ is in $\left\{I_{w_{j}^{\prime}}\right\}$; from Lemma 3 , there are now constants $C_{3}>0$ and $0<\mu_{3}<1$ such that

$$
\left|\log \left(\frac{s_{f}\left(w_{k_{0}} w_{n}\right)}{s_{f}\left(w_{k_{0}-l} w_{n}\right)}\right)\right| \leq C_{3} \mu_{3}^{n}
$$


Only case (2) remains. By applying Lemma 3, there are constants $C_{4}>0$ and $0<\mu_{4}<1$ such that

and

$$
\left|\log \left(\frac{s_{f}\left(b^{*}\right)}{s_{f}\left(w_{k_{0}} w_{n}\right)}\right)\right| \leq C_{4} \mu_{4}^{n}
$$

$$
\left|\log \left(\frac{s_{g}\left(b^{*}\right)}{s_{g}\left(w_{k_{0}} w_{n}\right)}\right)\right| \leq C_{4} \mu_{4}^{n} .
$$

Because $s_{g}=s_{f}$, for $C_{5}=2 C_{4}$ and $\mu_{5}=\mu_{4}$,

$$
\left|\log \left(\frac{s_{f}\left(w_{k_{0}} w_{n}\right)}{s_{g}\left(w_{k_{0}} w_{n}\right)}\right)\right| \leq C_{5} \mu_{5}^{n}
$$

Let $C_{0}$ be the minimum of the ratios $\log \left(\left|h\left(I_{w}\right)\right| /\left|I_{w}\right|\right)$ for $I_{w}$ in $\eta_{k_{0}}$. From the arguments above, there are constants $C>0$ and $0<\mu<1$ such that

$$
\left|\log \frac{\left|h\left(I_{w_{k_{0}} w_{n}}\right)\right|}{\left|I_{w_{k_{0}} w_{n}}\right|}\right| \leq C_{0}+C \sum_{i=1}^{n} \mu^{i} \leq C_{0}+\frac{C \mu}{1-\mu}
$$

for all $I_{w_{k_{0}} w_{n}}$ in $\eta_{k_{0}+n}$ and all $n \geq 0$. Hence there is a constant, still denoted $C>0$, such that

$$
C^{-1} \leq \frac{\left|h\left(I_{w_{k_{0}} w_{n}}\right)\right|}{\left|I_{w_{k_{0}} w_{n}}\right|} \leq C
$$

for all $I_{w_{k_{0}} w_{n}}$ in $\eta_{n+k_{0}}$ and all $n \geq 0$. Since the union of boundary points of all intervals in $\eta_{n+k_{0}}$ for all $n \geq 0$ is dense in $M$,

$$
C^{-1} \leq \frac{|h(x)-h(y)|}{|x-y|} \leq C
$$

for every pair $x$ and $y$ in $M$. In other words, $h$ is bi-Lipschitz continuous.

A geometrically finite one-dimensional map $f$ is mixing if for any interval $I$ in $\eta_{f}$, there is an integer $n>0$ such that $f^{\circ n}(I)=M$. Since the mixing condition is topologically invariant, we may speak of a topological conjugacy class $\mathcal{F}$ being mixing.

A singular point $p$ of $f$ is a fold singular point if $f^{\prime}(x) f^{\prime}(2 p-x)<0$ for $x \neq p$ near $p$. Let $F S P$ denote the set of all fold singular points of a geometrically finite onedimensional map $f$ and let $F S O=\bigcup_{i=1}^{\infty} f^{\circ i}(F S P)$ denote the post fold singular orbits. Let $\xi$ be the set of closures of intervals of $M \backslash F S O$.

Let $f$ be a geometrically finite one-dimensional map. Let $\eta$ be the natural Markov partition of $M$ to $f$. Let $\eta_{n}$ be the $n^{\text {th }}$ partition of $M$ induced by $f$ with $\eta$. Let $G S O=\bigcup_{i=0}^{\infty} \cup_{J \in \eta_{n}} \partial J$ be the grand singular orbits of $f$. For a mixing topological conjugacy class $\mathcal{F}$, we have the following rigidity result.

Theorem 4. Let $f$ and $g$ be maps in a mixing topological conjugacy class $\mathcal{F}$. Let $h$ be the conjugacy from $f$ to $g$, i.e., $h \circ f=g \circ h$. Then $h \mid I$ for any $I$ in $\xi$ is a $C^{1+\beta}$-diffeomorphism for some $0<\beta \leq 1$ if and only if (i) $h$ is differentiable at one point $p$ in $M \backslash G S O$ with non-zero derivative and (ii) the exponents and asymmetries of $f$ and $g$ at all corresponding critical points are the same.

We prove Theorem 4 by means of several lemmas. Let $\mathcal{F}$ be a mixing topological conjugacy class in the space of simple geometrically finite one-dimensional maps and let $f$ and $g$ be maps in $\mathcal{F}$. Suppose both $f$ and $g$ are $C^{1+\alpha}$ for some $0<\alpha \leq 1$. Let $h$ be the conjugacy from $f$ and $g: h \circ f=g \circ h$. Let $\eta$ be the natural Markov partition of $M$ to $f$. Let $\eta_{n}$ be the $n^{\text {th }}$ partition of $M$ induced by $f$ with $\eta$ for 
$0 \leq n<\infty$. Let $\Sigma^{*}$ be the dual phase space of $\mathcal{F}$. Let $C P=\left\{c_{1}, \cdots, c_{d}\right\}$ be the critical points of $f$. Let $\Gamma=\left\{\gamma_{1}, \cdots, \gamma_{d}\right\}$ be the set of corresponding exponents of $f$ at critical points and let $A S=\left\{A_{1}, A_{2}, \cdots, A_{d}\right\}$ be the set of corresponding asymmetries of $f$. Let $n_{0}$ and $U$ and $V$ be the fixed integer and the sets in $\S 3.1$ for $f$.

Suppose $I=I_{w_{n_{0}}} \in \eta_{n_{0}}$ and $a^{*}=\cdots w_{n} w_{n_{0}}$ is a point in $\Sigma^{*}$. Then $I_{w_{n} w_{n_{0}}}$ is an interval in $\eta_{n+n_{0}}$. For any $x$ and $y$ in $I$, let $x_{n}$ and $y_{n}$ in $I_{w_{n} w_{n_{0}}}$ be the preimage of $x$ and $y$ under $f^{\circ n}$ for $n \geq 0$.

Lemma 5. There is a constant $C>0$ such that if all $I_{w_{n} w_{n_{0}}}$ are in $V$, then

$$
\left|\log \left(\prod_{n=1}^{\infty} \frac{\left|f^{\prime}\left(y_{n}\right)\right|}{\left|f^{\prime}\left(x_{n}\right)\right|}\right)\right| \leq C \cdot|x-y|^{\alpha}
$$

and

$$
\left|\log \left(\prod_{n=1}^{\infty} \frac{\left|g^{\prime}\left(h\left(x_{n}\right)\right)\right|}{\left|g^{\prime}\left(h\left(y_{n}\right)\right)\right|}\right)\right| \leq C \cdot|h(x)-h(y)|^{\alpha} .
$$

Proof. From the proof of Lemma 3, there is a constant $C_{1}>0$ such that

$$
\left|\log \left(\prod_{i=1}^{\infty} \frac{\left|f^{\prime}\left(y_{i}\right)\right|}{\left|f^{\prime}\left(x_{i}\right)\right|}\right)\right| \leq C_{1} \cdot \sum_{i=1}^{\infty}\left|x_{i}-y_{i}\right|^{\alpha} .
$$

By applying Lemma 3 , we have a constant $C_{2}>0$ such that

$$
\frac{\left|x_{n}-y_{n}\right|}{\left|I_{w_{n+n_{0}}}\right|} \leq C_{2} \cdot \frac{|x-y|}{\left|I_{w_{n}}\right|}
$$

for all $1 \leq n<\infty$. So there are constants $C_{3}>0$ and $0<\mu_{1}<1$ such that

$$
\left|x_{n}-y_{n}\right| \leq C_{3} \cdot \mu_{1}^{n} \cdot|x-y|
$$

for all $0 \leq n<\infty$. This implies that

$$
\left|\log \left(\prod_{n=1}^{\infty} \frac{\left|f^{\prime}\left(y_{n}\right)\right|}{\left|f^{\prime}\left(x_{n}\right)\right|}\right)\right| \leq C \cdot|x-y|^{\alpha}
$$

for some constant $C>0$. Similarly,

$$
\left|\log \left(\prod_{n=1}^{\infty} \frac{\left|g^{\prime}\left(h\left(y_{n}\right)\right)\right|}{\left|g^{\prime}\left(h\left(x_{n}\right)\right)\right|}\right)\right| \leq C \cdot|h(x)-h(y)|^{\alpha} .
$$

Lemma 6. There is a constant $C>0$ such that if $I_{w_{n_{0}}}$ is in $U$, then

$$
\left|\log \left(\prod_{n=1}^{\infty} \frac{\left|f^{\prime}\left(y_{n}\right)\right|}{\left|f^{\prime}\left(x_{n}\right)\right|}\right)\right| \leq C \cdot|x-y|^{\alpha}
$$

and

$$
\left|\log \left(\prod_{n=1}^{\infty} \frac{\left|g^{\prime}\left(h\left(x_{n}\right)\right)\right|}{\left|g^{\prime}\left(h\left(y_{n}\right)\right)\right|}\right)\right| \leq C \cdot|h(x)-h(y)|^{\alpha}
$$


Proof. From the proof of Lemma 4 , there is a constant $C_{1}>0$ such that

$$
\left|\log \left(\prod_{n=1}^{\infty} \frac{\left|f^{\prime}\left(y_{n}\right)\right|}{\left|f^{\prime}\left(x_{n}\right)\right|}\right)\right| \leq C_{1} \cdot \sum_{n=1}^{\infty}\left|x_{n}-y_{n}\right|^{\alpha} .
$$

By applying Lemma 4 , we have a constants $C_{2}>0$ such that

$$
\frac{\left|x_{n}-y_{n}\right|}{\left|I_{w_{n+n_{0}}}\right|} \leq C_{2} \cdot \frac{|x-y|}{\left|I_{w_{n}}\right|}
$$

for all $1 \leq n<\infty$. So there are constant $C_{3}>0$ and $0<\mu_{1}<1$ such that

$$
\left|x_{n}-y_{n}\right| \leq C_{3} \cdot \mu_{1}^{n} \cdot|x-y|
$$

for all $0 \leq n<\infty$. This implies that

$$
\left|\log \left(\prod_{n=1}^{\infty} \frac{\left|f^{\prime}\left(y_{n}\right)\right|}{\left|f^{\prime}\left(x_{n}\right)\right|}\right)\right| \leq C \cdot|x-y|^{\alpha}
$$

for a constant $C>0$. Similarly,

$$
\left|\log \left(\prod_{n=1}^{\infty} \frac{\left|g^{\prime}\left(h\left(y_{n}\right)\right)\right|}{\left|g^{\prime}\left(h\left(x_{n}\right)\right)\right|}\right)\right| \leq C \cdot|h(x)-h(y)|^{\alpha} .
$$

Let $p$ be a point in $M \backslash G S O$. Consider the grand backward orbit of $p$ under $f, G O(p)=\bigcup_{i=0}^{\infty} f^{-i}(p)$. Then $G O(p)$ is a dense subset of $M$ since $f$ is mixing. From the equation $h \circ f=g \circ h$, we have that if $h^{\prime}(f(x)) \neq 0$ exists and if $x$ is not a singular point, then

$$
h^{\prime}(x)=\frac{f^{\prime}(x)}{g^{\prime}(h(x))} \cdot h^{\prime}(f(x)) \neq 0
$$

exists too. Therefore, if $h^{\prime}(p) \neq 0$ exits, then $h^{\prime}(x) \neq 0$ exists for any $x \in G O(p)$.

Lemma 7. If $h$ is differentiable at a point $p$ in $M \backslash G S O$ with non-zero derivative, then $h^{\prime} \mid G O(p)$ is continuous at $p$.

Proof. Since $h^{\prime}(p) \neq 0$ exists, there is an open interval $p \in W$ such that $h \mid W$ is bi-Lipschitz. Because the eigenvalue of an expanding periodic point of $f$ is a bi-Lipschitz invariant, we have

$$
\left(f^{\circ n}\right)^{\prime}(q)=\left(g^{\circ n}\right)^{\prime}(h(q))
$$

if $f^{\circ n}(q)=q \in W$.

Suppose $p \in I_{k}=I_{w_{k} w_{n_{0}}} \in \eta_{k+n_{0}}$ and that $I_{k} \subseteq W$. For any $x \in I_{k} \cap G O(p)$ with $f^{\circ n}(x)=p$, let $x_{i}=f^{\circ(n-i)}(x)$. Let $x_{i} \in I_{i+k} \in \eta_{i+k+n_{0}}$. Then $I_{n+k} \subseteq$ $f^{\circ n}\left(I_{n+k}\right)=I_{k}$. There is a point $q \in I_{n+k}$ such that $f^{\circ n}(q)=q$. From $h \circ f=g \circ h$, we have

$$
\frac{h^{\prime}(p)}{h^{\prime}(x)}=\frac{\left(g^{\circ n}\right)^{\prime}(h(x))}{\left(f^{\circ n}\right)^{\prime}(x)}=\frac{\left(g^{\circ n}\right)^{\prime}(h(x))}{\left(g^{\circ n}\right)^{\prime}(h(q))} \cdot \frac{\left(f^{\circ n}\right)^{\prime}(q)}{\left(f^{\circ n}\right)^{\prime}(x)} .
$$

Let $q_{i}=f^{\circ(n-i)}(q)$ and consider

$$
\frac{\left(f^{\circ n}\right)^{\prime}(q)}{\left(f^{\circ n}\right)^{\prime}(x)}=\prod_{i=1}^{n} \frac{f^{\prime}\left(q_{i}\right)}{f^{\prime}\left(x_{i}\right)} .
$$


If $I_{k} \subseteq U$, then from Lemma 6 ,

$$
\left|\log \left(\frac{\left|\left(f^{\circ n}\right)^{\prime}(q)\right|}{\left|\left(f^{\circ n}\right)^{\prime}(x)\right|}\right)\right| \leq C_{1}|p-q|^{\alpha} \leq C \mu_{1}^{k}
$$

where $C_{1}>0$ and $0<\mu_{1}<1$ are constants. Otherwise, consider the smallest integer $m>0$ such that $I_{m+k} \subseteq U_{j} \subset U$. From Lemma 5, there are constants $C_{2}>0$ and $0<\mu_{2}<1$ such that

$$
\left|\log \left(\prod_{i=1}^{m-1} \frac{\left|f^{\prime}\left(q_{i}\right)\right|}{\left|f^{\prime}\left(x_{i}\right)\right|}\right)\right| \leq C_{2}|p-q|^{\alpha} \leq C_{2} \mu_{2}^{k} .
$$

From Lemma 6 , there are constants $C_{3}>0$ and $0<\mu_{3}<1$ such that

$$
\left|\log \left(\prod_{i=m+1}^{n} \frac{\left|f^{\prime}\left(q_{i}\right)\right|}{\left|f^{\prime}\left(x_{i}\right)\right|}\right)\right| \leq C_{3}\left|x_{m}-q_{m}\right|^{\alpha} \leq C_{3} \mu_{3}^{k+m} .
$$

Because $f \mid U_{j}$ and $f^{\prime} \mid U_{j}$ are comparable to $\left|z-c_{j}\right|^{\gamma_{j}}+f\left(c_{j}\right)$ and $\left|z-c_{j}\right|^{\gamma_{j}-1}$, there are constants $C_{4}>0$ and $0<\mu_{4}<1$ such that

$$
\begin{aligned}
\left|\log \left(\frac{\left|f^{\prime}\left(q_{m}\right)\right|}{\left|f^{\prime}\left(x_{m}\right)\right|}\right)\right| & \leq C_{4}\left|x_{m}-q_{m}\right|^{\alpha}+\frac{1}{\gamma_{j}-1}\left|\log \left(\frac{\left|x_{m}-c_{j}\right|}{\left|q_{m}-c_{j}\right|}\right)\right| \\
& \leq C_{4} \mu_{4}^{m+k}+\frac{1}{\gamma_{j}-1}\left|\log \left(\frac{\left|x_{m}-c_{j}\right|}{\left|q_{m}-c_{j}\right|}\right)\right| .
\end{aligned}
$$

If the distance $d_{k}=\operatorname{dist}\left(I_{k}, P C O\right)$ between $I_{k}$ and $P C O$ is greater than the length $\left|I_{k}\right|$ of $I_{k}$, then $\left|x_{m}-q_{m}\right| \leq C_{6} \min \left\{\left|x_{m}-c_{j}\right|, q_{m}-c_{j} \mid\right\}$ and

$$
\begin{aligned}
\left|\log \left(\frac{\left|x_{m}-c_{j}\right|}{\left|q_{m}-c_{j}\right|}\right)\right| & \leq C_{7} \frac{\left|x_{m}-q_{m}\right|}{\left|q_{m}-c_{j}\right|} \leq C_{8}\left(\frac{\left|x_{m+1}-q_{m+1}\right|}{\left|q_{m+1}-f\left(c_{j}\right)\right|}\right)^{\frac{1}{\gamma_{j}}} \\
& \leq C_{9}\left(\frac{|p-q|}{d_{k}}\right)^{\frac{1}{\gamma_{j}}} \leq C_{10}\left(\frac{\mu_{10}^{k}}{d_{k}}\right)^{\frac{1}{\gamma_{j}}}
\end{aligned}
$$

where $C_{i}>0$ for $i=7,8,9,10$ and $0<\mu_{10}<1$ are constants. So we have constants $C>0$ and $0<\mu<1$ such that

$$
\left|\log \left(\frac{\left|\left(f^{\circ n}\right)^{\prime}(q)\right|}{\left|\left(f^{\circ n}\right)^{\prime}(x)\right|}\right)\right| \leq C\left(\mu^{k}+\mu^{k+m}+\frac{\mu^{k}}{d_{k}}\right) .
$$

Similarly, we can get

$$
\left|\log \left(\frac{\left|\left(g^{\circ n}\right)^{\prime}(h(x))\right|}{\left|\left(g^{\circ n}\right)^{\prime}(h(q))\right|}\right)\right| \leq C\left(\mu^{k}+\mu^{k+m}+\frac{\mu^{k}}{d_{k}^{\prime}}\right)
$$

where $d_{k}^{\prime}=\operatorname{dist}\left(h\left(I_{k}\right), h(P C O)\right)$. Thus

$$
\left|\log \frac{h^{\prime}(p)}{h^{\prime}(x)}\right| \leq 2 C\left(\mu^{k}+\mu^{k+m}+\frac{\mu^{k}}{\min \left\{d_{k}, d_{k}^{\prime}\right\}}\right) .
$$

This implies that $h^{\prime} \mid G O(p)$ is continuous at $p$.

Corollary 2. If $h$ is differentiable at a point $p$ in $M \backslash G S O$ with nonzero derivative, then $h^{\prime} \mid G O(p)$ is continuous at every point $x$ in $G O(p)$. 
Proof. We use the same notation as in Lemma 7. For any $x \in G O(p)$, let $f^{\circ n}(x)=$ $p$, let $p \in I_{k}=I_{w_{k} w_{n_{0}}} \in \eta_{k+n_{0}}$, and let $x \in I_{n+k} \in \eta_{n+k+n_{0}}$ such that $f^{\circ n}\left(I_{n+k}\right)=$ $I_{k}$. For any $y \in I_{n+k}$, we have

$$
\frac{h^{\prime}(x)}{h^{\prime}(y)}=\frac{\left(f^{\circ n}\right)^{\prime}(x)}{\left(f^{\circ n}\right)^{\prime}(y)} \cdot \frac{\left(g^{\circ n}\right)^{\prime}(h(y))}{\left(g^{\circ n}\right)^{\prime}(h(x))} \cdot \frac{h^{\prime}(p)}{h^{\prime}\left(f^{\circ n}(y)\right)} .
$$

As in the proof of Lemma 7 , we have

$$
\left|\log \left(\frac{h^{\prime}(x)}{h^{\prime}(y)}\right)\right| \leq C\left(\mu^{k}+\mu^{k+m}+\frac{\mu^{k}}{\min \left\{d_{k}, d_{k}^{\prime}\right\}}\right)+\left|\log \left(\frac{h^{\prime}(p)}{h^{\prime}\left(f^{\circ n}(y)\right)}\right)\right| .
$$

Now Lemma 7 implies that $h^{\prime} \mid G O(p)$ is continuous at $x$.

An interval $I_{w_{n_{0}}}$ in $\eta_{n_{0}}$ is called critical if one of its end-points is a critical point of $f$. Otherwise, it is called non-critical.

Lemma 8. If $h$ is differentiable at a point $p$ in $M \backslash G S O$ with non-zero derivative, then the restriction of $h$ to every critical interval in $\eta_{n_{0}}$ is $C^{1+\beta}$ for some $0<\beta \leq 1$.

Proof. Suppose $I$ is a critical interval in $\eta_{n_{0}}$. Then $I \subset U$. Since $f$ is mixing, there is a preimage $I_{n}$ of $I$ under $f^{\circ n}$ such that $I_{n}$ tends to $p$.

For any $x$ and $y$ in $I$, let $x_{n}$ and $y_{n}$ in $I_{n}$ be the preimages of $x$ and $y$ under $f^{\circ n}$. From the equation $h \circ f=g \circ h$, we have

Thus,

$$
\frac{h^{\prime}(x)}{h^{\prime}(y)}=\frac{\left(g^{\circ n}\right)^{\prime}\left(h\left(x_{n}\right)\right)}{\left(f^{\circ n}\right)^{\prime}\left(x_{n}\right)} \cdot \frac{\left(f^{\circ n}\right)^{\prime}\left(y_{n}\right)}{\left(g^{\circ n}\right)^{\prime}\left(h\left(y_{n}\right)\right)} \cdot \frac{h^{\prime}\left(x_{n}\right)}{h^{\prime}\left(y_{n}\right)} .
$$

$$
\begin{aligned}
\left|\log \left(\frac{h^{\prime}(x)}{h^{\prime}(y)}\right)\right| & \leq\left|\log \left(\frac{\left(g^{\circ n}\right)^{\prime}\left(h\left(x_{n}\right)\right)}{\left(g^{\circ n}\right)^{\prime}\left(h\left(y_{n}\right)\right)}\right)\right|+\left|\log \left(\frac{\left(f^{\circ n}\right)^{\prime}\left(y_{n}\right)}{\left(f^{\circ n}\right)^{\prime}\left(x_{n}\right)}\right)\right|+\left|\log \left(\frac{h^{\prime}\left(x_{n}\right)}{h^{\prime}\left(y_{n}\right)}\right)\right| \\
& \leq C\left(|x-y|^{\alpha}+|h(x)-h(y)|^{\alpha}\right)+\left|\log \left(\frac{h^{\prime}\left(x_{n}\right)}{h^{\prime}\left(y_{n}\right)}\right)\right| .
\end{aligned}
$$

Since $h^{\prime}\left(x_{n}\right) \rightarrow h^{\prime}(p)$ and $h^{\prime}\left(y_{n}\right) \rightarrow h^{\prime}(p)$ as $n$ tends to infinity, we have

$$
\left|\log \left(\frac{h^{\prime}(x)}{h^{\prime}(y)}\right)\right| \leq C\left(|x-y|^{\alpha}+|h(x)-h(y)|^{\alpha}\right) .
$$

This implies that $h^{\prime} \mid(I \cap G O(p))$ is uniformly continuous. Therefore, it can be extended to a continuous function $h^{\prime}$ on $I$. Furthermore, the last inequality implies again that $h$ is $C^{1+\beta}$ for $\beta=\alpha$.

Lemma 9. If $h$ is differentiable at a point $p$ in $M \backslash G S O$ with non-zero derivative and if the exponents of $f$ and $g$ at corresponding critical points are the same, then the restriction of $h$ to every interval $I \subseteq V$ in $\eta_{n_{0}}$ is $C^{1+\beta}$ for some $0<\beta \leq 1$.

Proof. Suppose $I_{n}$ in $\eta_{n_{0}+n}$ is a preimage of $I$ under $f^{\circ n}$. Since $f$ is mixing, we can choose $I_{n}$ such that $I_{n}$ tends to $p$. Let $I_{n, i}=f^{\circ(n-i)}\left(I_{n}\right)$ for $0 \leq i \leq n$.

For any $x$ and $y$ in $I$ and $n>0$, let $x_{n}$ and $y_{n}$ in $I_{n}$ be the preimages of $x$ and $y$ under $f^{\circ n}$. From the equation $h \circ f=g \circ h$, we have

$$
\frac{h^{\prime}(x)}{h^{\prime}(y)}=\frac{\left(g^{\circ n}\right)^{\prime}\left(h\left(x_{n}\right)\right)}{\left(f^{\circ n}\right)^{\prime}\left(x_{n}\right)} \cdot \frac{\left(f^{\circ n}\right)^{\prime}\left(y_{n}\right)}{\left(g^{\circ n}\right)^{\prime}\left(h\left(y_{n}\right)\right)} \cdot \frac{h^{\prime}\left(x_{n}\right)}{h^{\prime}\left(y_{n}\right)} .
$$

Thus,

$$
\left|\log \left(\frac{h^{\prime}(x)}{h^{\prime}(y)}\right)\right| \leq\left|\log \left(\frac{\left|\left(g^{\circ n}\right)^{\prime}\left(h\left(x_{n}\right)\right)\right|}{\left|\left(g^{\circ n}\right)^{\prime}\left(h\left(y_{n}\right)\right)\right|}\right)\right|+\left|\log \left(\frac{\left|\left(f^{\circ n}\right)^{\prime}\left(y_{n}\right)\right|}{\left|\left(f^{\circ n}\right)^{\prime}\left(x_{n}\right)\right|}\right)\right|+\left|\log \left(\frac{h^{\prime}\left(x_{n}\right)}{h^{\prime}\left(y_{n}\right)}\right)\right| .
$$


Let $m=m(n)>0$ be the smallest integer such that $I_{m, n} \subseteq U_{j} \subset U$. Then

$$
\begin{aligned}
\left|\log \left(\frac{h^{\prime}(x)}{h^{\prime}(y)}\right)\right| \leq & \left|\sum_{i=1}^{m-1}\left(\log \left|f^{\prime}\left(y_{i}\right)\right|-\log \left|f^{\prime}\left(x_{i}\right)\right|\right)\right| \\
& +\left|\sum_{i=1}^{m-1}\left(\log \left|g^{\prime}\left(h\left(x_{i}\right)\right)\right|-\log \left|g^{\prime}\left(h\left(y_{i}\right)\right)\right|\right)\right| \\
& +\left|\log \left(\frac{\left|g^{\prime}\left(h\left(x_{m}\right)\right)\right|}{\left|f^{\prime}\left(x_{m}\right)\right|} \cdot \frac{\left|f^{\prime}\left(y_{m}\right)\right|}{\left|g^{\prime}\left(h\left(y_{m}\right)\right)\right|}\right)\right| \\
& +\left|\sum_{i=m+1}^{n}\left(\log \left|f^{\prime}\left(y_{i}\right)\right|-\log \left|f^{\prime}\left(x_{i}\right)\right|\right)\right| \\
& +\left|\sum_{i=m+1}^{n}\left(\log \left|g^{\prime}\left(h\left(x_{i}\right)\right)\right|-\log \left|g^{\prime}\left(h\left(y_{i}\right)\right)\right|\right)\right| .
\end{aligned}
$$

From Lemma 5 , there is a constant $C_{1}>0$ such that

$$
\left|\sum_{i=1}^{m-1}\left(\log \left|f^{\prime}\left(y_{i}\right)\right|-\log \left|f^{\prime}\left(x_{i}\right)\right|\right)\right| \leq C_{1}|x-y|^{\alpha}
$$

and

$$
\left|\sum_{i=1}^{m-1}\left(\log \left|g^{\prime}\left(h\left(x_{i}\right)\right)\right|-\log \left|g^{\prime}\left(h\left(y_{i}\right)\right)\right|\right)\right| \leq C_{1}|h(x)-h(y)|^{\alpha} .
$$

From Lemma 6 , there are constants $C_{2}>0, C_{3}>0$, and $C_{4}>0$ such that

$$
\begin{aligned}
& \left|\sum_{i=m+1}^{n}\left(\log \left|f^{\prime}\left(y_{i}\right)\right|-\log \left|f^{\prime}\left(x_{i}\right)\right|\right)\right| \\
& \quad \leq C_{2}\left|x_{m}-y_{m}\right|^{\alpha} \leq C_{3}\left|x_{m-1}-y_{m-1}\right|^{\frac{\alpha}{\gamma_{j}}} \leq C_{4}|x-y|^{\frac{\alpha}{\gamma_{j}}} .
\end{aligned}
$$

Similarly,

$$
\begin{aligned}
& \left|\sum_{i=m+1}^{n}\left(\log \left|g^{\prime}\left(h\left(x_{i}\right)\right)\right|-\log \left|g^{\prime}\left(h\left(y_{i}\right)\right)\right|\right)\right| \\
& \quad \leq C_{2}\left|h\left(x_{m}\right)-h\left(y_{m}\right)\right|^{\frac{\alpha}{\gamma_{j}}} \leq C_{4}|h(x)-h(y)|^{\frac{\alpha}{\gamma_{j}}} .
\end{aligned}
$$

Now we consider

$$
S=\frac{\left|g^{\prime}\left(h\left(x_{m}\right)\right)\right|}{\left|f^{\prime}\left(x_{m}\right)\right|} \cdot \frac{\left|f^{\prime}\left(y_{m}\right)\right|}{\left|g^{\prime}\left(h\left(y_{m}\right)\right)\right|}
$$

Define

$$
S=S_{1} \cdot S_{2} \cdot S_{3}
$$

where

$$
\begin{gathered}
S_{1}=\frac{\left|g^{\prime}\left(h\left(x_{m}\right)\right)\right|}{\left|h\left(x_{m}\right)-h\left(c_{j}\right)\right|^{\gamma_{j}-1}} \cdot \frac{\left|h\left(y_{m}\right)-h\left(c_{j}\right)\right|^{\gamma_{j}-1}}{\mid g^{\prime}\left(h\left(y_{m}\right)\right)}, \\
S_{2}=\frac{\left|x_{m}-c_{j}\right|^{\gamma_{j}-1}}{\left|f^{\prime}\left(x_{m}\right)\right|} \cdot \frac{\left|f^{\prime}\left(y_{m}\right)\right|}{\left|y_{m}-c_{j}\right|^{\gamma_{j}-1}},
\end{gathered}
$$


and

$$
S_{3}=\left(\frac{\left|h\left(x_{m}\right)-h\left(c_{j}\right)\right|}{\left|x_{m}-c_{j}\right|}\right)^{\gamma_{j}-1} \cdot\left(\frac{\left|y_{m}-c_{j}\right|}{\left|h\left(y_{m}\right)-h\left(c_{j}\right)\right|}\right)^{\gamma_{j}-1} .
$$

Lemma 8 implies that

$$
\left|\log S_{3}\right| \leq C_{5}\left|x_{m}-y_{m}\right|^{\alpha} \leq C_{6}\left|x_{m-1}-y_{m-1}\right|^{\frac{\alpha}{\gamma_{j}}} \leq C_{7}|x-y|^{\frac{\alpha}{\gamma_{j}}}
$$

where $C_{i}$ for $i=5,6,7$ are constants. From (1) of Definition 3,

$$
\left|\log S_{2}\right| \leq C_{8}\left|x_{m}-y_{m}\right|^{\alpha} \leq C_{9}\left|x_{m-1}-y_{m-1}\right|^{\frac{\alpha}{\gamma_{j}}} \leq C_{10}|x-y|^{\frac{\alpha}{\gamma_{j}}}
$$

and

$$
\left|\log S_{1}\right| \leq C_{8}\left|h\left(x_{m}\right)-h\left(y_{m}\right)\right|^{\alpha} \leq C_{9}\left|h\left(x_{m-1}\right)-h\left(y_{m-1}\right)\right|^{\frac{\alpha}{\gamma_{j}}} \leq C_{10}|h(x)-h(y)|^{\frac{\alpha}{\gamma_{j}}}
$$

where $C_{i}$ for $i=8,9$, and 10 are constants. Thus,

$$
\left|\log \left(\frac{h^{\prime}(x)}{h^{\prime}(y)}\right)\right| \leq C\left(|x-y|^{\frac{\alpha}{\gamma}}+|h(x)-h(y)|^{\frac{\alpha}{\gamma}}\right)
$$

where $\gamma=\max \left\{\gamma_{i}\right\}_{i=1}^{d}$. So $h^{\prime} \mid(I \cap G O(p))$ is uniformly continuous. It can be extended to a continuous function on $I$. Furthermore, the last inequality implies that $h \mid I$ is $C^{1+\beta}$ for $\beta=\alpha / \gamma$.

Proof of Theorem 4. The "only if" part follows from direct calculation. Let us prove the "if" part. From Lemmas 8 and $9, h$ is $C^{1+\beta}$ for some $0<\beta \leq 1$ when it is restricted to any interval $I$ in $\eta_{n_{0}}$. Using the equation $h \circ f=g \circ h$, we have that $h$ is $C^{1+\beta}$ when it is restricted to any interval $I$ in the natural Markov partition $\eta$ of $M$ to $f$. It remains to check that $h$ is continuous on $I$ for any $I$ in $\xi$. If $s$ is a singular point, then we can find an open interval $W$ contained in an interval $I_{0}$ of $\eta_{0}$ such that $s \in f(W)$ and $f: W \rightarrow f(W)$ is a diffeomorphism. This implies that $h$ is continuous at $s$. If $s$ is not a fold singular point, we have, from the equation $h \circ f=g \circ h$, that for $x \neq s$ near $s$,

$$
h^{\prime}(f(x))=\frac{g^{\prime}(h(x))}{f^{\prime}(x)} h^{\prime}(x) .
$$

This implies that

$$
h^{\prime}(f(s)+)=\frac{B_{+, f}}{B_{+, g}} h^{\prime}(s) \quad \text { and } \quad h^{\prime}(f(s)-)=\frac{B_{-, f}}{B_{-, g}} h^{\prime}(s)
$$

where $B_{ \pm, f}$ and $B_{ \pm, g}$ are the numbers in the beginning of $\S 3$ for $f$ and $g$. Because $f$ and $g$ have the same asymmetry at $s$, the last equation implies that

$$
h^{\prime}(f(s)-)=h^{\prime}(f(s)+) .
$$

So $h$ is continuous at $f(s)$. Similarly, $h$ is continuous at all points $f^{\circ i}(s)$ for $i \geq 0$ if $s$ is not a fold singular point. Therefore, the restriction of $h$ to any interval $I$ in $\xi$ is $C^{1+\beta}$.

One consequence of Theorems 3 and 4 is that scaling functions together with exponents and asymmetries are complete $C^{1}$-invariants as follows: 
Theorem 5. Let $f$ and $g$ be maps in a mixing topological conjugacy class $\mathcal{F}$ and let $\Sigma^{*}$ be the dual space of $\mathcal{F}$. Let $h$ be the topological conjugacy from $f$ to $g$, i.e., $f \circ h=h \circ g$. Then $h \mid I$ for any $I$ in $\xi$ is a $C^{1+\beta}$-diffeomorphism for some $0<\beta \leq 1$ if and only if the scaling functions $s_{f}$ and $s_{g}$, on $\Sigma^{*}$, and the exponents and asymmetries of $f$ and $g$ at corresponding critical points are the same.

Proof. The "only if" part follows from Proposition 4, Remark 2 and direct calculation. Let us prove the "if" part. From Theorem $3, h$ is bi-Lipschitz. It is differentiable at almost all points in $M$. A bi-Lipschitz function is absolutely continuous; therefore, $h^{\prime}(x)>0$ exists for almost all points in $M$. Since $G S O$ has measure zero, there is at least one point $p$ in $M \backslash G S O$ such that $h^{\prime}(p)>0$ exists. Theorem 5 now follows from Theorem 4.

Remark 6. In [4] and [5] we have studied a special topological conjugacy class, which we call the space of Ulam-von Neumann transformations, of geometrically finite one-dimensional maps. We have shown that the set of eigenvalues at periodic points and the exponent and asymmetry at a unique critical point of a map in this space are complete $C^{1}$-invariants. This result can be also discussed for a mixing geometrically finite one-dimensional map.

\section{REFERENCES}

1. L. V. Ahlfors, Lectures on Quasiconformal Maps, Van Nostrand Company, Princeton, New Jersey, 1966. MR 34:336

2. M. Feigenbaum, Presentation functions, fixed points and a theory of scaling function dynamics, J. Statist. Phys. 52 (1988), 527-569. MR 90a:58112

3. M.-R. Herman, Sur la conjugaison différentiable des difféomorphismes du cercle à des rotations, Publ. Math. I. H. E. S., No. 49 (1979), 5-233. MR 81h:58039

4. Y. Jiang, Generalized Ulam-von Neumann transformations, Thesis, 1990, the Graduate School of CUNY.

5. Y. Jiang, On Ulam-von Neumann transformations, Commun. Math. Phys. 172 (1995), 449459.

6. Y. Jiang, Dynamics of certain smooth one-dimensional mappings - I. The $C^{1+\alpha}$-DenjoyKoebe distortion lemma, IMS preprint series 1991/1, SUNY at Stony Brook.

7. Y. Jiang, Geometry of geometrically finite one-dimensional maps, Commun. in Math. Phys. 156, 639-647, 1993. MR 95f:58033

8. R. de la Llave and R. Moriyón, Invariant for smooth conjugacy of hyperbolic dynamical systems. IV, Comm. in Math. Phys. 109, 369-378, 1987. MR 90h:58064

9. J. Milnor and W. Thurston, On iterated maps of the interval I and II, Preprint, Princeton University, 1977; finally published in Lecture Notes in Math. 1342 (1988), 465-563. MR 90a:58083

10. M. Shub and D. Sullivan, Expanding endomorphisms of the circle revisited, Ergod. Th \& Dynam. Sys., 5, 1987, 285-289. MR 87g:58104

11. D. Sullivan, Private conversation.

12. D. Sullivan, Differentiable structure on fractal-like sets determined by intrinsic scaling functions on dual Cantor sets, Proceedings of Symposia in Pure Mathematics, Vol. 48, 1988, 15-23. MR 90k:58141

Department of Mathematics, Queens College of CUnY, Flushing, New York 11367

E-mail address: yungc@yunping.math.qc.edu

E-mail address: yungc@qcunix.acc.qc.edu 